\title{
WHITE NOISE APPROACH TO STOCHASTIC INTEGRATION
}

\author{
L. ACCARDI*-W. AYED**-H. OUERDIANE***
}

\begin{abstract}
We extend to white noise integrals the scalar type integrator inequalities introduced by Accardi, Fagnola and Quaegebeur [1] as a generalization of the Hudson-Parthasarathy basic estimates on stochastic integrals. We use these estimates as "regularity results", showing that some Hida distributions are in fact elements of the Fock space. We also use them to prove an analogue regularity result for solutions of white noise equations with bounded coefficients.
\end{abstract}

The white noise approach to stochastic calculus emerged, between 1993 and 1995, from the stochastic limit of quantum theory as a new approach [7] to both classical and quantum stochastic calculus [9], [16]. The main achievements of the new approach have been:

(i) The identification of both classical and quantum stochastic equations with white noise Hamiltonian equations.

(ii) The explanation of the emergence of the unitarity conditions of Hudson and Parthsarathy as expression of the symmetricity of the associated Hamiltonian equation.

(iii) The explicit expression of the coefficients of the stochastic equation as (nonlinear) functions of the coefficients of the associated Hamiltonian equation.

(iv) The emergence of a natural nonlinear extension of stochastic calculus. The deep and surprising results obtained in this direction in the quadratic case suggest that the completion of this programme for the higher powers of white noise is one of the most challenging and fascinating problems of contemporary stochastic analysis.

None of these results could have been even formulated in the framework of the usual (classical or quantum) stochastic analysis. However the exciting new developments emerged from the white noise approach to stochastic calculus delayed a systematic exposition of its basic analytical tools such as white noise integrals, white noise equations, the theory of distributions on the standard simplex, the causal normal order, ...

A large literature existed in the first two of the above mentioned directions, in the framework of Hida's white noise analysis. However to create a bridge between these results and those of classical and quantum stochastic analysis, one needs some "regularity results", i.e. conditions which assure that objects, which a priori are Hida distributions, are in fact vectors in a Hilbert space. Such regularity conditions will be formulated here in terms of estimates on white noise integrals.

Estimates of this type were developed in [3] and they were strong enough to prove an existence theorem for a multidimensional white noise integral equation. However, as we shall see in a forthcoming paper [8], these estimates were not strong enough to prove the fundamental result of the theory, i.e. the unitarity condition. The main results of the present paper are:

(i): The extension of the white noise estimates of [3] to a larger domain (the "maximal algebraic" domain introduced in Section 1) on the lines of [5].

(ii): The introduction of the notion of white noise adaptedness and the proof, under this assumption (which requires a white noise with 1-dimensional parameter), of the white noise analogue of the "scalar type integrator" inequalities of Accardi, Fagnola and Quaegebeur [1] which, in their turn, generalize the basic Hudson-Parthasarathy estimate on stochastic integrals (cf. Proposition 25.6 in [15]).

Key words and phrases. White noise-Maximal algebraic domain, Estimates on white noise stochastic integrals.

*:Università di Roma Tor Vergata, Centro Vito Volterra 00133 Rome, Italy. Mail: accardi volterra.mat.uniroma2.it.

**:University of Tunis El Manar, Department of Mathematics 1060 Tunis, Tunisia. Mail: wided.ayed ipein.rnu.tn.

***:University of Tunis El Manar, Department of Mathematics 1060 Tunis, Tunisia. Mail: habib.ouerdiane fst.rnu.tn. AMS classification: $60 \mathrm{H} 40-60 \mathrm{H} 15$. 
The present paper is the first step of our plan to complete the programme initiated in [3] of giving a systematic derivation of the white noise unitarity conditions. Here we only deal with the basic estimates on stochastic integrals and with the corresponding existence theorem for stochastic differential equations.

\section{NOTATIONS}

In this section, in order to fix our notations, we review some well known material. The terms quantum white noise and free field are synonyms: the former is more used in mathematics, the latter in physics. Since classical white noise is included in quantum white noise, in the following we will use the term white noise to mean the more general (quantum) case. A standard way to construct white noises is through the Fock space. We will consider the scalar Boson Fock space over $L^{2}\left(\mathbb{R}^{d}\right)$ :

$$
\begin{gathered}
\mathcal{F}\left(L^{2}\left(\mathbb{R}^{d}\right)\right):=\mathcal{F}:=\bigoplus_{n=o}^{\infty} L_{\text {sym }}^{2}\left(\mathbb{R}^{d n}\right)=\oplus_{n=0}^{\infty} \mathcal{F}_{n} \quad ; \quad \mathcal{F}_{0}:=\mathbb{C} \\
\mathcal{F}_{n}:=L_{\text {sym }}^{2}\left(\mathbb{R}^{d n}\right), n=1,2, \ldots
\end{gathered}
$$

is the Hilbert space of square integrable functions of $n$-variables in $\mathbb{R}^{d}$, symmetric under the permutation of their arguments. If $S$ is a subspace of $L^{2}\left(\mathbb{R}^{d}\right)$ we will use the notation

$$
\mathcal{F}(S):=\bigoplus_{n=o}^{\infty} \otimes_{\text {sym }}^{n} S
$$

which is clearly a subspace of $\mathcal{F}\left(L^{2}\left(\mathbb{R}^{d}\right)\right)$ because, for each $n, \otimes_{\text {sym }}^{n} S \subseteq \mathcal{F}_{n}$. The elements of $\mathcal{F}_{n}$ are called $n$-particle vectors and the set of $n$-particle vectors, for all $n \in \mathbb{N}$, is also called the set of number vectors. For an element $\psi^{(n)} \in \mathcal{F}_{n}$ we write $\psi^{(n)}=\psi^{(n)}\left(s_{1}, \ldots, s_{n}\right), s_{i} \in \mathbb{R}^{d}$ and, for any permutation $\pi$, on $\{1, \ldots, n\}$ one has:

$$
\psi^{(n)}\left(s_{1}, \ldots, s_{n}\right)=\psi^{(n)}\left(s_{\pi(1)}, \ldots, s_{\pi(n)}\right) .
$$

So an element of the Boson Fock space $\mathcal{F}$ is a sequence of functions $\psi=\left\{\psi^{(0)}, \psi^{(1)}, \psi^{(2)}, \ldots.\right\}$ where $\psi^{(0)} \in \mathbb{C}, \psi^{(n)} \in \mathcal{F}_{n}, n=1,2, \ldots$ and

$$
\|\psi\|^{2}=\sum_{n=0}^{\infty}\left\|\psi^{(n)}\right\|_{L^{2}\left(\mathbb{R}^{d n}\right)}^{2}<\infty
$$

More explicitly

$$
\|\psi\|^{2}=\left|\psi^{(0)}\right|^{2}+\sum_{n=1}^{\infty} \int_{R^{d n}}\left|\psi^{(n)}\left(s_{1}, \ldots, s_{n}\right)\right|^{2} d s_{1} \ldots d s_{n}
$$

The inner product of elements $\psi=\left\{\psi^{(n)}\right\}_{n=0}^{\infty}$ and $\phi=\left\{\phi^{(n)}\right\}_{n=0}^{\infty}$ in $\mathcal{F}$ is given by

$$
(\psi, \phi)=\sum_{n=0}^{\infty}\left(\psi^{(n)}, \phi^{(n)}\right)=\overline{\psi^{(0)}} \phi^{(0)}+\sum_{n=1}^{\infty} \int_{R^{d n}} \overline{\psi^{(n)}\left(s_{1}, \ldots, s_{n}\right)} \phi^{(n)}\left(s_{1}, \ldots, s_{n}\right) d s_{1} \ldots d s_{n}
$$

The vector $\Phi=(1,0,0, \ldots)$ is called the vacuum vector. A vector $\psi=\left(\psi^{(n)}\right)$ such that there exists $f \in L^{2}\left(\mathbb{R}^{d}\right)$ with the property that, $\forall n \in \mathbb{N}$, we have

$$
\begin{gathered}
\psi^{(n)}\left(s_{1}, \cdots, s_{n}\right)=\frac{1}{\sqrt{n !}} f\left(s_{1}\right) f\left(s_{2}\right) \cdots f\left(s_{n}\right) ; \text { a.e for } n \geq 1 \\
\psi^{(0)}=1 \Phi \\
\psi_{0}=\Phi
\end{gathered}
$$

is called an exponential vector with test function $f$ and denoted $\psi_{f}$ or $\psi(f)=\left(\psi_{f}^{(n)}\right)$. 
Definition 0.1. Let $S\left(\mathbb{R}^{d n}\right)$ denote the Schwartz space, i.e. the set of infinitely differentiable complex valued functions $h$ on $\mathbb{R}^{\text {nd }}$ such that, for any $\alpha, \beta \in \mathbb{N}^{\text {nd }}$ one has:

$$
\|h\|_{\alpha, \beta}=\sup _{x \in \mathbb{R}^{n d}}\left|x^{\alpha} D^{(\beta)} h(x)\right|<\infty
$$

Define the subspaces of $\mathcal{F}$ :

$$
\begin{gathered}
\mathcal{F}\left(\mathcal{S}\left(\mathbb{R}^{d}\right)\right):=\mathcal{D}_{S}:=\left\{\psi \in \mathcal{F}: \forall n \in \mathbb{N}, \psi^{(n)} \in S\left(\mathbb{R}^{d n}\right)\right\} \\
\mathcal{N}:=\left\{\psi \in \mathcal{D}_{S}: \psi^{(n)}=0 \text { for almost all } n \in \mathbb{N}\right\} \\
\mathcal{D}_{S}^{0}:=\mathcal{N} \cap \mathcal{D}_{S}
\end{gathered}
$$

These are the vectors in $\mathcal{F}$ for which all components, with the exception of at most a finite number are equal to zero. They are called finite particle vectors. We also define:

$$
\begin{aligned}
\mathcal{D}_{S}^{1} & :=\left\{\psi \in \mathcal{D}_{S}: \sum_{n=1}^{\infty} n\left\|\psi^{(n)}\right\|^{2}<\infty\right\} \\
\mathcal{D}^{1} & :=\left\{\psi \in \mathcal{F}: \sum_{n=1}^{\infty} n\left\|\psi^{(n)}\right\|^{2}<\infty\right\}
\end{aligned}
$$

Similarly we define $\mathcal{D}_{C}, \mathcal{D}_{C}^{0}, \mathcal{D}_{C}^{1}$ by replacing in (1.1.a), (1.1.b), (1.1.c) the Schwartz space $S\left(\mathbb{R}^{n d}\right)$ with the space $C\left(\mathbb{R}^{n d}\right) \cap L^{2}\left(\mathbb{R}^{n d}\right)$ where $C\left(\mathbb{R}^{n d}\right)$ denotes the space of continuous functions on $\mathbb{R}^{n d}$.

Remark 0.1. Each of the 3 spaces $\mathcal{D}_{S}, \mathcal{D}_{S}^{0}, \mathcal{D}_{S}^{1}$ is dense in $\mathcal{F}$.

Definition 0.2. For any $s \in \mathbb{R}^{d}, n \in \mathbb{N}$ and for any $\psi \in \mathcal{D}^{1}$ we fix a representative $\psi^{(n)}\left(s_{1}, \cdots, \cdots, s_{n}\right)$ in the Lebesgue class of $\psi^{(n)}$ and we define:

a-: the annihilation density $a_{s}$ as the linear operator:

$$
a_{s}: \psi \in \mathcal{D}^{1} \rightarrow\left(a_{s} \psi\right)^{(n)}\left(s_{1}, s_{2}, \cdots, s_{n}\right)=\sqrt{n+1} \psi^{(n+1)}\left(s, s_{1}, s_{2}, \cdots, s_{n}\right) \in \mathcal{F}
$$

which associates to $\psi \in \mathcal{D}^{1}$ the $\mathcal{F}$-valued linear functional on the square integrable functions on $\mathbb{R}^{d}$ :

$$
f \mapsto \sqrt{n+1} \int d s f(s) \psi^{(n+1)}\left(s, s_{1}, s_{2}, \cdots, s_{n}\right)=:\left(A_{f} \psi\right)^{(n)}\left(s_{1}, \cdots, s_{n}\right)
$$

$A_{f}$ is called the annihilation operator. It follows that

$$
A_{f} \psi=\int_{\mathbb{R}^{d}} a_{s} f(s) \psi d s .
$$

b-: the creation density $a_{s}^{+}$as the linear operator:

$$
a_{s}^{+}: \psi \in \mathcal{F} \rightarrow\left(a_{s}^{+} \psi\right)^{(n)}\left(s_{1}, s_{2}, \cdots, s_{n}\right)=\frac{1}{\sqrt{n}} \sum_{i=1}^{n} \delta\left(s-s_{i}\right) \psi^{(n-1)}\left(s_{1}, \cdots, \hat{s}_{i}, \cdots, s_{n}\right)
$$

which associates to $\psi \in \mathcal{D}^{1}$ the $\mathcal{F}$-valued linear functional on the square integrable functions on $\mathbb{R}^{d}$ :

$$
f \mapsto \frac{1}{\sqrt{n}} \sum_{i=1}^{n} f\left(s_{i}\right) \psi^{(n-1)}\left(s_{1}, \cdots, \hat{s}_{i}, \cdots, s_{n}\right)=:\left(A_{f}^{+} \psi\right)^{(n)}\left(s_{1}, \cdots, s_{n}\right)
$$

$A_{f}^{+}$is called the creation operator and we get:

$$
\begin{aligned}
\left(A_{f}^{+} \psi\right)^{(n)}\left(s_{1}, \cdots, s_{n}\right) & =\frac{1}{\sqrt{n}} \sum_{i=1}^{n} \int_{\mathbb{R}^{d}} \delta\left(s-s_{i}\right) f(s) \psi^{(n-1)}\left(s_{1}, \cdots, \hat{s}_{i}, \cdots, s_{n}\right) d s \\
& =\int_{\mathbb{R}^{d}}\left(a_{s}^{+} \psi\right)^{(n)} f(s) d s .
\end{aligned}
$$


It follows that

$$
A_{f}^{+} \psi=\int_{\mathbb{R}^{d}} a_{s}^{+} f(s) \psi d s
$$

c-: the number density is defined on $\mathcal{D}^{1}$ by:

$$
n_{s}=a_{s}^{+} a_{s}
$$

Using (den1) and (den2), for any $\psi \in \mathcal{D}^{1}$, one has

$$
\left(n_{s} \psi\right)^{(n)}\left(s_{1}, s_{2}, \cdots, s_{n}\right)=\sum_{i=1}^{n} \delta\left(s-s_{i}\right) \psi^{(n)}\left(s, s_{1}, \cdots, \hat{s}_{i}, \cdots, s_{n}\right)
$$

which allows to interpret the number density in the same way as the creation density.

Remark 0.2. Taking $f=\chi_{[0, t]}$ in 0.3 and 0.6 , we obtain:

$$
A_{t}=\int_{o}^{t} a_{s} d s
$$

and

$$
A_{t}^{+}=\int_{o}^{t} a_{s}^{+} d s
$$

These notations are those for white noise over $\mathbb{R}, a_{t}, a_{t}^{+}$, since a Brownian motion ( classical or quantum ) is obtained by integrating a white noise. Also, we get [4] the commutation relations:

$$
\begin{gathered}
{\left[A_{t}, A_{s}^{+}\right]=\min (s, t) ;\left[A_{t}, A_{s}\right]=0=\left[A_{t}^{+}, A_{s}^{+}\right]} \\
A_{t} \Phi=0
\end{gathered}
$$

Proposition 0.1. The operators introduced in Definition (0.2) are well defined and depend only on the sequence $\left(\psi^{(n)}\right)$ (and not on the representatives of its elements). Moreover

$$
\begin{gathered}
A_{f}: \mathcal{F}_{n} \longrightarrow \mathcal{F}_{n-1} \quad ; \quad A_{f} \mathcal{F}_{0}=0, n=1,2, \cdots \\
A_{f}^{+}: \mathcal{F}_{n} \longrightarrow \mathcal{F}_{n+1}, \quad n=0,1,2, \cdots
\end{gathered}
$$

The creation and the annihilation operators are adjoint to each other on $\mathcal{D}^{1}$, and we have:

$$
A_{f} \Phi=0
$$

Proof. For any measurable function $\psi^{(n)}$ the right hand side of (den1) is well defined and the identity

$$
\left\langle\varphi^{(n-1)},\left(A_{f} \psi\right)^{(n-1)}\right\rangle=\int_{\mathbb{R}^{d n}} \overline{f\left(s_{n}\right)} \tilde{\psi}^{(n)}\left(s_{1}, \cdots, s_{n}\right) \varphi^{(n-1)}\left(s_{1}, \cdots, s_{n-1}\right) d s_{1} \cdots d s_{n}
$$

shows that the Lebesgue class of $\left(A_{f} \psi\right)^{(n-1)}$ does not depend on the choice of $\psi^{(n)}\left(s_{1}, \cdots, s_{n}\right)$ but only on its Lebesgue class $\psi^{(n)}$. The fact that $\psi=\left(\psi^{(n)}\right) \in \mathcal{D}^{1}$ and the Schwartz inequality the sequence $\left(\left(A_{f} \psi\right)^{(n)}\right)$ defines a continuous linear functional on $\mathcal{F}$ hence a unique element in $\mathcal{F}$. If $\psi \in \mathcal{D}^{1}$ and $f \in L^{2}\left(\mathbb{R}^{d}\right), A_{f}^{+} \psi$ is in $\mathcal{F}$ because:

$$
\begin{gathered}
\left\|A_{f}^{+} \psi\right\|^{2}=\sum_{n=1}^{\infty} \int_{R^{d n}}\left|\left(A_{f}^{+} \psi\right)^{(n)}\left(s_{1}, \ldots, s_{n}\right)\right|^{2} d s_{1} \ldots d s_{n} \\
=\sum_{n=1}^{\infty} \int_{R^{d n}} \mid\left(\left.\frac{1}{\sqrt{n}} \sum_{i=1}^{n} f\left(s_{i}\right) \psi^{(n-1)}\left(s_{1}, \cdots, \hat{s}_{i}, \cdots, s_{n}\right)\right|^{2} d s_{1} \ldots d s_{n}\right. \\
=\sum_{n=1}^{\infty} \int_{R^{d n}} n\left|f\left(s_{1}\right) \psi^{(n-1)}\left(s_{2}, \cdots, s_{n}\right)\right|^{2} d s_{1} \ldots d s_{n}
\end{gathered}
$$




$$
=\|f\|^{2}\left\{\sum_{n=1}^{\infty} n\left\|\psi^{(n-1)}\right\|_{L^{2}\left(\mathbb{R}^{(n-1) d}\right)}^{2}\right\}<\infty
$$

The same argument used above shows that the Lebesgue class of $\left(a_{f}^{+}\right)^{(n)}$ only depends on the classes of $f$ and $\psi^{(n-1)}$. The remaining statements are proved in a similar way.

Definition 0.3. We can extend the definition of number operator; let $T \hat{\in} B\left(L^{2}\left(\mathbb{R}^{d}\right)\right)$ be a pre-closed linear operator with integral kernel $\tau$, i.e.for all $f \in L^{2}\left(\mathbb{R}^{d}\right)$ :

$$
T f(x)=\int \tau(x, y) f(y) d y
$$

The number operator $N_{T}$ is defined by:

$$
N_{T}=\iint \tau(x, y) a_{x}^{+} a_{y} d x d y
$$

Notice that, for all $f \in \operatorname{Dom}(T) \subseteq L^{2}\left(\mathbb{R}^{d}\right)$ and for all exponential vectors $\psi_{f}$, one has:

$$
N_{T} \psi_{f}=\int d x \int d y \tau(x, y) f(y) a_{x}^{+} \psi_{f}=\int d x(T f)(x) a_{x}^{+} \psi_{f}=A_{T f}^{+} \psi_{f} .
$$

We allow $\tau(x, y)$ to be a distribution: the choice

$$
\tau(x, y)=V(x) \delta(x-y)
$$

allows to include all the multiplication operators.

Remark 0.3. The Boson commutation relations:

$$
\left[a_{s_{1}}, a_{s_{2}}^{+}\right]=\delta\left(s_{1}-s_{2}\right)
$$

are interpreted weakly on $\mathcal{D}^{1}$ and easily verified on that domain.

\section{The Maximal Algebraic Domain}

$\mathcal{D}^{1}$ is not an invariant domain under the action of all creation, annihilation, number and Weyl operators. There is a number of invariant domains which are useful in different situations [9].

In this section we introduce the smallest domain containing the vacuum and invariant under the action of all these operators. We call it "maximal algebraic domain" that is the largest domain obtainable from the vacuum with purely algebraic operations on the basic operators.

Definition 1.1. The maximal algebraic domain denoted by $\mathcal{D}_{M A D}$ is by definition the linear span of the vectors set

$$
\mathcal{D}_{M A D}^{0}=\left\{A_{f_{n}}^{+} \cdots A_{f_{1}}^{+} \psi_{f}, \psi_{f}, / f, f_{1}, \cdots, f_{n} \in L^{2}\left(\mathbb{R}^{d}\right), n \geq 1\right\}
$$

where $A_{f}^{+}$for $f \in L^{2}\left(\mathbb{R}^{d}\right)$ is the creator operator, and $\psi_{f}$ is the exponential vectors with test function $f$.

Since $\mathcal{D}_{M A D}$ contains the exponential vectors it is dense in $\mathcal{F}$.

Lemma 1.1. For every $f_{0}, f_{1}, \cdots, f_{n} \in L^{2}\left(\mathbb{R}^{d}\right)$ one has $\psi_{f_{0}} \in \operatorname{Dom}\left(A_{f_{n}}^{+} \cdots A_{f_{1}}^{+}\right) ; \forall n \geq 1$.

Proof. We will prove by induction on $n \geq 1$, that for any $f_{0}, f_{1}, \cdots, f_{n} \in L^{2}\left(\mathbb{R}^{d}\right)$, one has $A_{f_{n}}^{+} \cdots A_{f_{1}}^{+} \psi_{f_{0}} \in \mathcal{F}$, so for $n=1$, since for all $f, g \in L^{2}\left(\mathbb{R}^{d}\right)$, we have (see [3]) :

$$
\left[A_{f}, A_{g}^{+}\right]=\langle f, g\rangle
$$


it follows:

$$
\begin{aligned}
\left\|A_{f_{1}}^{+} \psi_{f_{0}}\right\|^{2} & =\left\langle A_{f_{1}}^{+} \psi_{f_{0}}, A_{f_{1}}^{+} \psi_{f_{0}}\right\rangle \\
& =\left\langle\psi_{f_{0}}, A_{f_{1}} A_{f_{1}}^{+} \psi_{f_{0}}\right\rangle \\
& =\left\|f_{1}\right\|^{2}\left\|\psi_{f_{0}}\right\|^{2}+\left\langle\psi_{f_{0}}, A_{f_{1}}^{+} A_{f_{1}} \psi_{f_{0}}\right\rangle \\
& =\left\|f_{1}\right\|^{2}\left\|\psi_{f_{0}}\right\|^{2}+\left\langle A_{f_{1}} \psi_{f_{0}}, A_{f_{1}} \psi_{f_{0}}\right\rangle \\
& =\left\|f_{1}\right\|^{2}\left\|\psi_{f_{0}}\right\|^{2}+\left|\left\langle f_{1}, f_{0}\right\rangle\right|^{2}\left\|\psi_{f_{0}}\right\|^{2}<\infty
\end{aligned}
$$

Suppose that for any $f_{0}, f_{1}, \cdots, f_{n} \in L^{2}\left(\mathbb{R}^{d}\right)$, we have $A_{f_{n}}^{+} \cdots A_{f_{1}}^{+} \psi_{f_{0}} \in \mathcal{F}$ and let $f_{0}, f_{1}, \cdots, f_{n+1} \in$ $L^{2}\left(\mathbb{R}^{d}\right)$. To prove that $A_{f_{n+1}}^{+} A_{f_{n}}^{+} \cdots A_{f_{1}}^{+} \psi_{f_{0}} \in \mathcal{F}$, we will use the fact:

$$
A_{f_{n+1}} A_{f_{n+1}}^{+} A_{f_{n}}^{+} \cdots A_{f_{1}}^{+} \psi_{f_{0}}=\left\langle f_{n+1}, f_{0}\right\rangle A_{f_{n}}^{+} \cdots A_{f_{1}}^{+} \psi_{f_{0}}+\sum_{j=1}^{n+1}\left\langle f_{n+1}, f_{j}\right\rangle A_{f_{n+1}}^{+} \cdots \hat{A_{f_{j}}} \cdots A_{f_{1}}^{+} \psi_{f_{0}} \in \mathcal{F}
$$

then:

$$
\begin{aligned}
& \left\|A_{f_{n+1}}^{+} \cdots A_{f_{1}}^{+} \psi_{f_{0}}\right\|^{2} \\
= & \left\langle A_{f_{n}}^{+} \cdots A_{f_{1}}^{+} \psi_{f_{0}}, A_{f_{n+1}} A_{f_{n+1}}^{+} \cdots A_{f_{1}}^{+} \psi_{f_{0}}\right\rangle \\
= & \left\langle f_{n+1}, f_{0}\right\rangle\left\|A_{f_{n}}^{+} \cdots A_{f_{1}}^{+} \psi_{f_{0}}\right\|^{2}+\sum_{j=1}^{n+1}\left\langle A_{f_{n}}^{+} \cdots A_{f_{1}}^{+} \psi_{f_{0}}, A_{f_{n+1}}^{+} \cdots \hat{A_{f_{j}}} \cdots A_{f_{1}}^{+} \psi_{f_{0}}\right\rangle\left\langle f_{n+1}, f_{j}\right\rangle \\
\leq & \left(\max _{j=0, \cdots, n+1}\left\|f_{j}\right\|^{2}\right)\left\|A_{f_{n}}^{+} \cdots A_{f_{1}}^{+} \psi_{f_{0}}\right\|\left(\left\|A_{f_{n}}^{+} \cdots A_{f_{1}}^{+} \psi_{f_{0}}\right\|+\sum_{j=1}^{n+1}\left\|A_{f_{n+1}}^{+} \cdots \hat{A_{f_{j}}} \cdots A_{f_{1}}^{+} \psi_{f_{0}}\right\|\right)<\infty
\end{aligned}
$$

Now, to prove some property of invariance of $\mathcal{D}_{M A D}$, we will recall the definition of the Fock-Weyl representation of $L^{2}\left(\mathbb{R}^{d}\right)$ given in [3], in the following:

the Weyl operator $W_{f}$ is an element of the unitary group $U(\mathcal{F})$ with the strong operators topology. Moreover $W_{f}$ acts on the exponential vector $\psi_{g}$ as:

$$
W_{f}\left(\psi_{g}\right)=e^{-\frac{1}{2}\|f\|^{2}-\langle f, g\rangle} \psi(g+f) .
$$

we also have the following properties

1-:

$$
W_{g} \Phi=e^{-\frac{1}{2}\|g\|^{2}} \psi_{g}
$$

2-:

or equivalently

$$
\left(W_{g}\right)^{*} A_{f} W_{g}=A_{f}+\frac{i}{\sqrt{2}}\langle f, g\rangle
$$

$$
\left[A_{f}, W_{g}\right]=\frac{i}{\sqrt{2}}\langle f, g\rangle W_{g}
$$

Lemma 1.2. For every $g, f_{1}, \cdots, f_{n} \in L^{2}\left(\mathbb{R}^{d}\right)$ and $T \hat{\in} B\left(L^{2}\left(\mathbb{R}^{d}\right)\right)$, the linear span of the vectors $A_{f_{n}}^{+} \cdots A_{f_{1}}^{+} \psi_{g}$ is the smallest vector subspace of $\mathcal{F}$ containing the vacuum vector and invariant under the action of the operators

$$
A_{f}^{+}, A_{f}, W_{h}, N_{T}
$$

where $W_{h}, N_{T}$ are the Weyl and the number operators. Moreover, on the domain $\mathcal{D}_{M A D}$, one has:

$$
\begin{gathered}
\left(A_{f}^{+}\right)^{*}=A_{f} \\
\left(N_{T}\right)^{*}=N_{T^{*}}
\end{gathered}
$$


Proof. Let $g, f_{1}, \cdots, f_{n} \in L^{2}\left(\mathbb{R}^{d}\right)$, using (1.2.a), the prove of the fact that $A_{f} A_{f_{n}}^{+} \cdots A_{f_{1}}^{+} \psi_{g} \in$ $\mathcal{D}_{M A D}$ follows from

$$
A_{f} A_{f_{n}}^{+} \cdots A_{f_{1}}^{+} \psi_{g}=\langle f, g\rangle A_{f_{n}}^{+} \cdots A_{f_{1}}^{+} \psi_{g}+\sum_{j=1}^{n}\left\langle f, f_{j}\right\rangle A_{f_{n}}^{+} \cdots \hat{A_{f_{j}}} \cdots A_{f_{1}}^{+} \psi_{g} \in \mathcal{D}_{M A D} .
$$

To prove that $W_{f} A_{f_{n}}^{+} \cdots A_{f_{1}}^{+} \psi_{g} \in \mathcal{D}_{M A D}$, it is sufficient to prove that $W_{f} A_{f_{n}}^{+} \cdots A_{f_{1}}^{+} W_{g} \Phi \in \mathcal{D}_{M A D}$. Hence

$$
\begin{aligned}
W_{f} A_{f_{n}}^{+} \cdots A_{f_{1}}^{+} W_{g} \Phi & =W_{f} A_{f_{n}}^{+} \cdots A_{f_{1}}^{+} W_{f}^{*} W_{f} W_{g} \Phi \\
& =\prod_{i=1}^{n}\left[W_{f} A_{f_{i}}^{+} W_{f}^{*}\right] W_{f} W_{g} \Phi \\
& =\prod_{i=1}^{n}\left[A_{f_{i}}^{+}+\frac{i}{\sqrt{2}}\left\langle f, f_{i}\right\rangle\right] e^{i I m\langle f, g\rangle} W_{(f+g)} \Phi \\
& =\sum_{\left\{j_{1}, \cdots, j_{\alpha}\right\} \subset\{1, \cdots, n\}} \lambda_{\alpha}\left[A_{f_{j_{1}}}^{+} \cdots A_{f_{j_{\alpha}}}^{+} W_{(f+g)} \Phi\right] \in \mathcal{D}_{M A D}
\end{aligned}
$$

where

$$
\lambda_{\alpha}:=\left(-\frac{i}{\sqrt{2}}\right)^{n-\alpha} \prod_{\left\{h_{1}, \ldots, h_{n-\alpha}\right\}=\left\{j_{1}, \ldots, j_{\alpha}\right\}^{c} \subseteq\{1, \ldots, n\}}\left\langle f, f_{i}\right\rangle
$$

Let $f, f_{1} \in\left(L^{2}\left(\mathbb{R}^{d}\right)\right)$, then using

$$
\left[N_{T}, A_{f}^{+}\right]=A_{(T f)}^{+}, \quad N_{T} \psi_{f}=A_{T f}^{+} \psi_{f}
$$

we obtain:

$$
N_{T} A_{f_{1}}^{+} \psi_{f}=A_{f_{1}}^{+} N_{T} \psi_{f}+A_{T_{f_{1}}}^{+} \psi_{f}=A_{f_{1}}^{+} A_{T f}^{+} \psi_{f}+\left\langle T_{f_{1}}, f\right\rangle \psi_{f} \in \mathcal{D}_{M A D}
$$

by induction, suppose that for $f, g, f_{1}, \cdots, f_{n+1}$ belongs to $\in L^{2}\left(\mathbb{R}^{d}\right)$ one has

$$
N_{T} A_{f_{n}}^{+} \cdots A_{f_{1}}^{+} \psi_{g} \in \mathcal{D}_{M A D}
$$

and using (1.3), we get:

$$
N_{T} A_{f_{n+1}}^{+} A_{f_{n}}^{+}, \cdots A_{f_{1}}^{+} \psi_{g}=A_{f_{n+1}}^{+} N_{T} A_{f_{n}}^{+} \cdots A_{f_{1}}^{+} \psi_{g}+A_{\left(T f_{n+1}\right)}^{+} A_{f_{n}}^{+} \cdots A_{f_{1}}^{+} \psi_{g} \in \mathcal{D}_{M A D}
$$

Corollary 1.1. Denote by $\mathcal{P}_{W}$ the *algebra generated by the operators (1.2) acting on $\mathcal{D}_{M A D}$ which called the polynomial-Weyl algebra. We have:

$$
\mathcal{D}_{M A D}=\mathcal{P}_{W} \Phi
$$

Proof. It is clear that

$$
\mathcal{D}_{M A D} \subseteq \mathcal{P}_{W} \Phi
$$

The converse inclusion, i.e:

$$
\mathcal{P}_{W} \Phi \subseteq \mathcal{D}_{M A D}
$$

follows easily from the relations (1.2.a), (1.2.b) and (0.9). 


\section{White NOISE STOCHASTIC INTEGRALS}

In this section we will discuss white noise and stochastic integrals in $\mathbb{R}^{d}$ rather than in $\mathbb{R}$ because exactly the same formulae are valid in the 1-and in the d-dimensional case, but, as we shall see in Section (3), some estimates are slightly worse in the non adapted case, compared to the will be called white noise adapted. We define the operators:

$$
A(F)=<F, A>=\int_{\mathbb{R}^{d}} d s \bar{F}_{s} a_{s} ; A^{+}(F)=<A^{+}, F>=\int_{\mathbb{R}^{d}} d s a_{s}^{+} F_{s}
$$

where $F$ is a complex valued function on $\mathbb{R}^{d}$. The generalization of these integrals to the case when $F$ in an operator valued function are called right (resp. left) stochastic integrals with respect to $a_{s}$ $\left(\right.$ resp. $a_{s}^{+}$). Similarly one defines the two-sided stochastic integral:

$$
\int_{\mathbb{R}^{d}} d s a_{s}^{+} F_{s} a_{s}
$$

It is clear that, the existence of left and two sided stochastic integral, (also of right ones if the integrand process $F_{s}$ is unbounded), requires some compatibility conditions on the domains.

Definition 2.1. Let $\mathcal{L}:=\mathcal{L}(D)$ be the space of maps

$$
\begin{aligned}
F: \mathbb{R}^{d} & \rightarrow \mathcal{L}(D, \mathcal{F}) \\
s & \mapsto F_{s}
\end{aligned}
$$

where $\mathcal{L}(D, \mathcal{F})$ is the space of linear operators on the Fock space $\mathcal{F}$ densely defined on a domain $D$, such that for any $\varphi, \psi \in \mathcal{D}$, the maps

$$
s \in \mathbb{R}^{d} \mapsto<\psi, F_{s} \varphi>; s \mapsto\left\|F_{s} \psi\right\|^{2} \varphi, \psi \in D
$$

are locally integrable.

Elements of $\mathcal{L}$ will be called processes or, if confusion may arise, $D$-processes.

Remark 2.1. If $D=\mathcal{D}_{M A D}$, then the map $s \mapsto a_{s}$ is in $\mathcal{L}$, while the map $s \mapsto a_{s}^{+}$is not in $\mathcal{L}$

\subsection{Right annihilator stochastic integral.}

Definition 2.2. The right annihilator stochastic integral of $F \in \mathcal{L}$ is the operator:

$$
\psi \mapsto \int_{\mathbb{R}^{d}} d s F_{s} a_{s} \psi
$$

where the integral is meant as a Bochner integral in the Fock space. It is defined for each $\psi \in \mathcal{F}$ such that $a_{s} \psi$ is in the domain of $F_{s}$ for each $s$ and the vector valued function $s \in \mathbb{R}^{d} \mapsto F_{s} a_{s} \psi$ is Bochner integrable [14].

Lemma 2.1. The stochastic integral (2.1.a) is defined for all processes $F \in \mathcal{L}$ and for all vectors $\psi \in \mathcal{D}_{C}^{1}$ such that

$$
\sum_{n \geq 0} \sqrt{n}\left\|\int_{\mathbb{R}^{d}} d s F_{s} \psi^{(n)}(s, .)\right\|<\infty
$$

In this case the integral (2.1.a) is equal to

$$
\int_{\mathbb{R}^{d}} F_{s} a_{s} d s \psi=\sum_{n \geq 0} \sqrt{n+1} \int_{\mathbb{R}^{d}} d s F_{s} \psi^{(n+1)}(s, .)
$$

where $\psi^{(n+1)}(s,$.$) is the function$

$$
\left(s_{1}, \ldots, s_{n}\right) \in \mathbb{R}^{d n} \mapsto \psi^{(n+1)}\left(s, s_{1}, \ldots, s_{n}\right)
$$


Proof. We start from the explicit form of the right annihilator on a vector $\psi \in \mathcal{D}_{C}^{1}$

$$
\left(a_{s} \psi\right)^{(n)}=\sqrt{n+1} \psi^{(n+1)}(s, .)
$$

Therefore, in the notation (2.1)

$$
\int_{\mathbb{R}^{d}} F_{s} a_{s} \psi d s=\int_{\mathbb{R}^{d}} d s \sum_{n \geq 0} \sqrt{n+1} F_{s} \psi^{(n+1)}(s, .)
$$

Now by assumption, for each $n$, the function

$$
s \in \mathbb{R}^{d} \mapsto F_{s} \psi^{(n+1)}(s, .)
$$

is Bochner integrable. Moreover, because of (2.1.b), the series on the right hand side of (2.1.c) is absolutely convergent. Therefore, one can exchange the series and the integral. This gives (2.1.d).

If $\psi=\psi_{f}$; an the exponential vectors, the explicit form of the stochastic integral (2.1.a) is

$$
\int_{\mathbb{R}^{d}} d s F_{s} a_{s} \psi_{f}=\int_{\mathbb{R}^{d}} d s f(s) F_{s} \psi_{f}
$$

where the right hand side of (2.2) is defined on the set of the exponential vectors $\psi_{f}$ with test function in $L^{2}\left(\mathbb{R}^{d}\right)$ such that the vector valued function $s \mapsto f(s) F_{s} \psi_{f}$ is Bochner integrable.

\subsection{The left creator stochastic integral.}

Definition 2.3. The left creator stochastic integrals of a measurable element $F \in \mathcal{L} ; F: s \mapsto F_{s}$ is the operator:

$$
\psi \mapsto \int_{\mathbb{R}^{d}} d s a_{s}^{+} F_{s} \psi
$$

and it is given by the formula for the scalar case:

$$
\left(\int_{\mathbb{R}^{d}} d s a_{s}^{+} F_{s} \psi\right)^{(n)}\left(s_{1}, \ldots, s_{n}\right)=\frac{1}{\sqrt{n}} \sum_{i=1}^{n}\left(F_{s_{i}} \psi\right)^{(n-1)}\left(s_{1}, \ldots, \widehat{s_{i}}, \ldots, s_{n}\right), \forall n \geq 1
$$

where $\psi$ is in the domain of the operator $F_{s}$ for all $s \in \mathbb{R}_{+}$.

Remark 2.2. This definition has a meaning for any measurable function $s \mapsto F_{s}$, the natural domain of the left creator stochastic integral is

$$
\left\{\psi: \sum_{n=1}^{\infty}\left\|\left(\int_{\mathbb{R}^{d}} d s a_{s}^{+} F_{s} \psi\right)^{(n)}\right\|_{L^{2}\left(\mathbb{R}^{n d}\right)}^{2}<\infty\right\}
$$

or more explicitly, a vector $\psi$ in $\mathcal{D}\left(\int_{\mathbb{R}^{d}} d s a_{s}^{+} F_{s}\right)$ if and only if $\psi \in D\left(F_{s}\right), \forall s \in \mathbb{R}^{d}$

$$
\sum_{n=1}^{\infty} n \int_{\mathbb{R}^{d n}}\left|\left(F_{s_{1}} \psi\right)^{(n-1)}\left(s_{2}, \ldots, s_{n}\right)\right|^{2} d s_{1} \ldots d s_{n}<\infty
$$

We want now to obtain estimates on the norm of $\int_{\mathbb{R}^{d}} d s a_{s}^{+} F_{s} \psi$ which guarantees that the stochastic integral exist. An example of such estimates is given by the following lemma:

Lemma 2.2. Let $\psi$ belong to $\mathcal{D}\left(F_{s}\right)$ for all $s \in \mathbb{R}^{d}$. Then one has, for each $n \in \mathbb{N}^{*}$

$$
\left\|\left(\int_{\mathbb{R}^{d}} d s a_{s}^{+} F_{s} \psi\right)^{(n)}\right\|^{2} \leq n \int_{\mathbb{R}^{d}} d s\left\|\left(F_{s} \psi\right)^{(n-1)}\right\|^{2}
$$


Proof We have

$$
\begin{aligned}
& \left\|\left(\int d s a_{s}^{+} F_{s} \psi\right)^{(n)}\right\|^{2} \\
= & \int \ldots \int d s_{1} \ldots d s_{n} \frac{1}{n} \sum_{i, j}\left\langle\left(F_{s_{i}} \psi\right)^{(n-1)}\left(s_{1}, \ldots, \widehat{s_{i}}, \ldots, s_{n}\right),\left(F_{s_{j}} \psi\right)^{(n-1)}\left(s_{1}, \ldots, \widehat{s_{j}}, \ldots, s_{n}\right)\right\rangle \\
\leq & \frac{1}{n} \sum_{i, j} \int \ldots \int d s_{1} \ldots d s_{n}\left\|\left(F_{s_{i}} \psi\right)^{(n-1)}\left(s_{1}, \ldots, \widehat{s_{i}}, \ldots, s_{n}\right)\right\|\left\|\left(F_{s_{j}} \psi\right)^{(n-1)}\left(s_{1}, \ldots, \widehat{s_{j}}, \ldots, s_{n}\right)\right\| \\
= & \frac{n^{2}}{n} \int_{\mathbb{R}^{n}} d s \int_{\mathbb{R}^{d(n-1)}} d s_{2} \ldots d s_{n}\left\|\left(F_{s} \psi\right)^{(n-1)}\left(s_{2}, \ldots, s_{n}\right)\right\|^{2}=n \int_{\mathbb{R}^{d}} d s\left\|\left(F_{s} \psi\right)^{(n-1)}\right\|^{2}
\end{aligned}
$$

\subsection{The normally ordered two-sided integral.}

Definition 2.4. In the above notations, the two-sided normally ordered integral of $F \in \mathcal{L}$ :

$$
\int_{\mathbb{R}^{d}} d s a_{s}^{+} F_{s} a_{s}
$$

is defined,weakly on the maximal algebraic domain:

$$
\forall \xi, \eta \in \mathcal{D}_{M A D},\left\langle\xi, \int_{\mathbb{R}^{d}} d s a_{s}^{+} F_{s} a_{s} \eta\right\rangle=\int_{\mathbb{R}^{d}} d s\left\langle a_{s} \xi, F_{s} a_{s} \eta\right\rangle
$$

In particular, on exponential vectors one has

$$
\left\langle\psi_{f}, \int_{\mathbb{R}^{d}} d s a_{s}^{+} F_{s} a_{s} \psi_{g}\right\rangle=\left(\int_{\mathbb{R}^{d}} d s \bar{f}(s) g(s)\right)\left\langle\psi_{f}, F_{s} \psi_{g}\right\rangle
$$

Lemma 2.3. For any $n \in \mathbb{N}$ and for any exponential vector $\psi_{f}$ one has the estimate

$$
\left\|\left(\int_{\mathbb{R}^{d}} d s a_{s}^{+} F_{s} a_{s} \psi_{f}\right)^{(n)}\right\|^{2} \leq n \int_{\mathbb{R}^{d}} d s|f(s)|^{2}\left\|\left(F_{s} \psi_{f}\right)^{(n-1)}\right\|^{2}
$$

\section{Multidimensional estimates on $\mathcal{D}_{M A D}$}

In this section, we prove some estimates on the maximal algebraic domain in the multidimensional case. Since $\mathcal{D}_{M A D}$ is linearly spanned by the vectors of the form $A_{f_{n}}^{+} \cdots A_{f_{1}}^{+} \psi_{f}$, then it is useful to have estimates on the norm of these vectors. Recall that $\mathcal{F}=\Gamma\left(L^{2}\left(\mathbb{R}^{d}\right)\right)$ the symmetric Fock space over $L^{2}\left(\mathbb{R}^{d}\right)$ and $\mathcal{D}_{M A D}(C)$ is the maximal algebraic domain i.e. the linear span of the vectors in the set

$$
\mathcal{D}_{C}=\left\{A_{f_{n}}^{+} \cdots A_{f_{1}}^{+} \psi_{f}, \psi_{f}, / f, f_{1}, \cdots, f_{n} \in C=L^{2}\left(\mathbb{R}^{d}\right) \cap C\left(\mathbb{R}^{d}\right), n \geq 1\right\}
$$

Lemma 3.1. We have: a)

$$
\left[a_{s}, A_{f}^{+}\right]=f(s)
$$

where $f$ is a test function in $L^{2}\left(\mathbb{R}^{d}\right)$.

b)

$$
\left[a_{s}, A_{f_{n}}^{+} \cdots A_{f_{1}}^{+}\right]=\sum_{i=1}^{n} f_{i}(s) A_{f_{n}}^{+} \cdots \hat{A}_{f_{i}}^{+} \cdots A_{f_{1}}^{+}
$$

where $f_{1}, \cdots, f_{n}$ are test functions in $L^{2}\left(\mathbb{R}^{d}\right)$.

Proof. a) For all $\psi \in \mathcal{D}_{M A D}$ We have:

$$
\left(A_{f}^{+}(\psi)\right)^{(n)}\left(s_{1}, \cdots, s_{n}\right)=\frac{1}{\sqrt{n}} \sum_{i=1}^{n} f\left(s_{i}\right) \psi^{(n-1)}\left(s_{1}, \cdots, \hat{s}_{i}, \cdots, s_{n}\right)
$$


Using that:

$$
\left(a_{s} \psi\right)^{(n)}\left(s_{1}, \cdots, s_{n}\right)=\sqrt{n+1} \psi^{(n+1)}\left(s, s_{1}, \cdots, s_{n}\right)
$$

we obtain:

$$
\begin{aligned}
\left(a_{s} A_{f}^{+}(\psi)\right)^{(n)}\left(s_{1}, \cdots, s_{n}\right) & =\sqrt{n+1}\left(A_{f}^{+}(\psi)\right)^{(n+1)}\left(s, s_{1}, \cdots, s_{n}\right) \\
& =\frac{\sqrt{n+1}}{\sqrt{n+1}} \sum_{i=1}^{n+1} f\left(s_{i}\right) \psi^{(n)}\left(s_{1}, \cdots, \hat{s}_{i}, \cdots, s_{n+1}\right) \\
& =\sum_{i=1}^{n+1} f\left(s_{i}\right) \psi^{(n)}\left(s_{1}, \cdots, \hat{s}_{i}, \cdots, s_{n+1}\right)
\end{aligned}
$$

where $s_{n+1}=s$. Moreover:

$$
\begin{aligned}
\left(A_{f}^{+} a_{s} \psi\right)^{(n)}\left(s_{1}, \cdots, s_{n}\right) & =\frac{1}{\sqrt{n}} \sum_{i=1}^{n} f\left(s_{i}\right)\left(a_{s} \psi\right)^{(n-1)}\left(s_{1}, \cdots, \hat{s}_{i}, \cdots, s_{n}\right) \\
& =\frac{1}{\sqrt{n}} \sum_{i=1}^{n} f\left(s_{i}\right) \sqrt{n}(\psi)^{(n)}\left(s, s_{1}, \cdots, \hat{s}_{i}, \cdots, s_{n-1}\right)
\end{aligned}
$$

then:

$$
\begin{gathered}
\left(\left(a_{s} A_{f}^{+} \psi\right)^{(n)}-\left(A_{f}^{+} a_{s} \psi\right)^{(n)}\right)\left(s_{1}, \cdots, s_{n}\right)=\left(\left(a_{s} A_{f}^{+} \psi\right)^{(n)}-\left(A_{f}^{+} a_{s} \psi\right)^{(n)}\right)\left(s_{1}, \cdots, s_{n}\right) \\
=\sum_{i=1}^{n+1} f\left(s_{i}\right) \psi^{(n)}\left(s_{1}, \cdots, \hat{s}_{i}, \cdots, s_{n+1}\right)-\sum_{i=1}^{n} f\left(s_{i}\right)(\psi)^{(n)}\left(s, s_{1}, \cdots, \hat{s}_{i}, \cdots, s_{n-1}\right) \\
=f\left(s_{n+1}\right) \psi^{(n)}\left(s_{1}, \cdots, s_{n}\right) \\
=f(s) \psi^{(n)}\left(s_{1}, \cdots, s_{n}\right)
\end{gathered}
$$

b) This result will be proved by induction on $n$ : let $\mathcal{P}_{n}$ be the following property:

$$
\left[a_{s}, A_{f_{n}}^{+} \cdots A_{f_{1}}^{+}\right]=\sum_{i=1}^{n} f_{i}(s) A_{f_{n}}^{+} \cdots \hat{A}_{f_{i}}^{+} \cdots A_{f_{1}}^{+} .
$$

Using a) the case $n=1$ was verified, so we suppose that $\mathcal{P}_{n}$ was verified, then we will prove $\mathcal{P}_{n+1}$ so:

$$
\begin{aligned}
{\left[a_{s}, A_{f_{n+1}}^{+} \cdots A_{f_{1}}^{+}\right] } & =a_{s} A_{f_{n+1}}^{+} \cdots A_{f_{1}}^{+}-A_{f_{n+1}}^{+} \cdots A_{f_{1}}^{+} a_{s} \\
& =\left[a_{s}, A_{f_{n+1}}^{+}\right] A_{f_{n}}^{+} \cdots A_{f_{1}}^{+}+A_{f_{n+1}}^{+} a_{s} A_{f_{n}}^{+} \cdots A_{f_{1}}^{+}-A_{f_{n+1}}^{+} \cdots A_{f_{1}}^{+} a_{s} \\
& =\left[a_{s}, A_{f_{n+1}}^{+}\right] A_{f_{n}}^{+} \cdots A_{f_{1}}^{+}+A_{f_{n+1}}^{+}\left[a_{s}, A_{f_{n}}^{+} \cdots A_{f_{1}}^{+}\right] \\
& =f_{n+1}(s) A_{f_{n}}^{+} \cdots A_{f_{1}}^{+}+A_{f_{n+1}}^{+} \sum_{i=1}^{n} f_{i}(s) A_{f_{n}}^{+} \cdots \hat{A}_{f_{i}}^{+} \cdots A_{f_{1}}^{+} \\
& =\sum_{i=1}^{n+1} f_{i}(s) A_{f_{n+1}}^{+} \cdots \hat{A}_{f_{i}}^{+} \cdots A_{f_{1}}^{+}
\end{aligned}
$$

Proposition 3.1. (Right annihilator estimates)

For each $F \in \mathcal{L}$ and for each bounded subset $I \subseteq \mathbb{R}^{d}$, it follows : 
i): for each number vector: $\psi=A_{f_{n}}^{+} \cdots A_{f_{1}}^{+} \Phi$ where $f_{1}, \cdots, f_{n}$ are test functions in $L^{2}\left(\mathbb{R}^{d}\right)$, we have:

$$
\left\|\int_{I} d s F_{s} a_{s} \psi\right\| \leq c_{\psi, I} \sum_{\varphi \in J(\psi)}\left(\int_{I} d s\left\|F_{s} \varphi\right\|^{2}\right)^{\frac{1}{2}}
$$

where

$$
\begin{gathered}
J(\psi):=\left\{A_{f_{n}}^{+} \cdots \hat{A}_{f_{i}}^{+} \cdots A_{f_{1}}^{+} \Phi, / 1 \leq i \leq n\right\} \subset \mathcal{D}_{M A D} \\
c_{\psi, I}:=\max _{1 \leq i \leq n}\left(\left\|f_{i}\right\|_{2, I}\right) \\
\left\|f_{i}\right\|_{2, I}:=\left(\int_{I}\left|f_{i}(s)\right|^{2} d s\right)^{\frac{1}{2}}
\end{gathered}
$$

ii): For each vector $\psi=A_{f_{n}}^{+} \cdots A_{f_{1}}^{+} \psi_{f}$ and for $f, f_{1}, \cdots, f_{n}, I \subseteq \mathbb{R}^{d}$ (as in (i) above), (3.3) hold with

$$
\begin{gathered}
J(\psi)=\left\{A_{f_{n}}^{+} \ldots \hat{A}_{f_{i}}^{+} \ldots A_{f_{1}}^{+} \psi_{f}, A_{f_{n}}^{+} \ldots A_{f_{1}}^{+} \psi_{f}, 1 \leq i \leq n\right\} \\
c_{\psi, I}=\max _{1 \leq i \leq n}\left(\left\|f_{i}\right\|_{2, I},\|f\|_{2, I}\right)
\end{gathered}
$$

Proof i) Using lemma (3.1) and the fact that $a_{s} \Phi=0$, we have for $f_{1}, \cdots, f_{n} \in L^{2}\left(\mathbb{R}^{d}\right)$ :

$$
\begin{aligned}
\int_{I} d s F_{s} a_{s} A_{f_{n}}^{+} \cdots A_{f_{1}}^{+} \Phi & =\int_{I} d s F_{s}\left[a_{s}, A_{f_{n}}^{+} \cdots A_{f_{1}}^{+}\right] \Phi \\
& =\int_{I} d s F_{s} \sum_{i=1}^{n} f_{i}(s) A_{f_{n}}^{+} \cdots \hat{A_{f_{i}}^{+} \cdots A_{f_{1}}^{+} \Phi}
\end{aligned}
$$

so

$$
\begin{aligned}
\left\|\int_{I} d s F_{s} a_{s} A_{f_{n}}^{+} \cdots A_{f_{1}}^{+} \Phi\right\| & =\left\|\int_{I} d s F_{s} \sum_{i=1}^{n} f_{i}(s) A_{f_{n}}^{+} \cdots \hat{A}_{f_{i}}^{+} \cdots A_{f_{1}}^{+} \Phi\right\| \\
& \leq \sum_{i=1}^{n} \int_{I} d s\left|f_{i}(s)\right|\left\|F_{s} A_{f_{n}}^{+} \cdots \hat{A_{f_{i}}^{+}} \cdots A_{f_{1}}^{+} \Phi\right\| \\
& \leq \sum_{i=1}^{n}\left(\int_{I} d s\left|f_{i}(s)\right|^{2}\right)^{\frac{1}{2}}\left(\int_{I} d s\left\|F_{s} A_{f_{n}}^{+} \cdots \hat{A_{f_{i}}^{+}} \cdots A_{f_{1}}^{+} \Phi\right\|^{2}\right)^{\frac{1}{2}} \\
& \leq c_{\psi, I} \sum_{i=1}^{n}\left(\int_{I} d s\left\|F_{s} A_{f_{n}}^{+} \cdots \hat{A_{f_{i}}^{+}} \cdots A_{f_{1}}^{+} \Phi\right\|^{2}\right)^{\frac{1}{2}}
\end{aligned}
$$

and this proves (3.3) where $c_{\psi, I}$ is given by (3.1.b).

ii)

For $\psi=A_{f_{n}}^{+} \cdots A_{f_{1}}^{+} \psi_{f}$, where $f, f_{1}, \cdots, f_{n}$ are test functions and $\psi_{f}$ is an exponential vector, we have:

$$
\begin{aligned}
\int_{I} d s F_{s} a_{s} A_{f_{n}}^{+} \cdots A_{f_{1}}^{+} \psi_{f} & =\int_{I} d s F_{s}\left[a_{s}, A_{f_{n}}^{+} \cdots A_{f_{1}}^{+}\right] \psi_{f}+\int_{I} d s F_{s} f(s) A_{f_{n}}^{+} \cdots A_{f_{1}}^{+} \psi_{f} \\
& =\int_{I} d s F_{s}\left[\sum_{i=1}^{n} f_{i}(s) A_{f_{n}}^{+} \cdots{\hat{A_{f}}}^{+} \cdots A_{f_{1}}^{+} \psi_{f}+f(s) A_{f_{n}}^{+} \cdots A_{f_{1}}^{+} \psi_{f}\right]
\end{aligned}
$$


So:

$$
\begin{aligned}
& \left\|\int_{I} d s F_{s} a_{s} A_{f_{n}}^{+} \cdots \hat{A}_{f_{i}}^{+} \cdots A_{f_{1}}^{+} \psi_{f}\right\|=\left\|\int_{I} d s F_{s}\left[\sum_{i=1}^{n} f_{i}(s) A_{f_{n}}^{+} \cdots \hat{A}_{f_{i}}^{+} \cdots A_{f_{1}}^{+} \psi_{f}+f(s) A_{f_{n}}^{+} \cdots A_{f_{1}}^{+} \psi_{f}\right]\right\| \\
& \leq \int_{I} d s\left\|\left[\sum_{i=1}^{n} F_{s} f_{i}(s) A_{f_{n}}^{+} \cdots \hat{A}_{f_{i}}^{+} \cdots A_{f_{1}}^{+} \psi_{f}+F_{s} f(s) A_{f_{n}}^{+} \cdots A_{f_{1}}^{+} \psi_{f}\right]\right\| \\
& \leq \int_{I} d s\left[\left\|\sum_{i=1}^{n} F_{s} f_{i}(s) A_{f_{n}}^{+} \cdots \hat{A}_{f_{i}}^{+} \cdots A_{f_{1}}^{+} \psi_{f}\right\|+\left\|F_{s} f(s) A_{f_{n}}^{+} \cdots A+_{f_{1}} \psi_{f}\right\|\right] \\
& \leq \int_{I} d s\left[\sum_{i=1}^{n}\left|f_{i}(s)\right|\left\|F_{s} A_{f_{n}}^{+} \cdots \hat{A}_{f_{i}}^{+} \cdots A_{f_{1}}^{+} \psi_{f}\right\|+|f(s)|\left\|F_{s} A_{f_{n}}^{+} \cdots A_{f_{1}}^{+} \psi_{f}\right\|\right] \\
& \leq \sum_{i=1}^{n}\left(\int_{I}\left|f_{i}(s)\right|^{2} d s\right)^{\frac{1}{2}}\left(\int_{I} d s\left\|F_{s} A_{f_{n}}^{+} \cdots \hat{A}_{f_{i}}^{+} \cdots A_{f_{1}}^{+} \psi_{f}\right\|^{2}\right)^{\frac{1}{2}} \\
& +\left(\int_{I}|f(s)|^{2} d s\right)^{\frac{1}{2}}\left(\int_{I} d s\left\|F_{s} A_{f_{n}}^{+} \cdots A_{f_{1}}^{+} \psi_{f}\right\|^{2}\right)^{\frac{1}{2}} \\
& \leq c_{\psi, I} \sum_{i=1}^{n}\left(\int_{I} d s\left\|F_{s} A_{f_{n}}^{+} \cdots \hat{A}_{f_{i}}^{+} \cdots A_{f_{1}}^{+} \psi_{f}\right\|^{2}\right)^{\frac{1}{2}} \\
& +\left(\int_{I} d s\left\|F_{s} A_{f_{n}}^{+} \cdots A_{f_{1}}^{+} \psi_{f}\right\|^{2}\right)^{\frac{1}{2}}
\end{aligned}
$$

where $c_{\psi, I}$ is as in $(3.1 . \mathrm{d})$

\subsection{Multidimensional left creator estimate.}

Lemma 3.2. Consider the stochastic integral

$$
A_{I}^{+}(F) \psi:=\int_{I} d s a_{s}^{+} F_{s} \psi, I \subseteq \mathbb{R}^{d}
$$

where $F \in \mathcal{L}$ and $\forall s \in I, \psi \in \operatorname{Dom}\left(F_{s}\right)$ such that

$$
\sum_{n=1}^{\infty} n \int_{\mathbb{R}^{d n}}\left|\left(F_{s_{1}} \psi\right)^{(n-1)}\left(s_{2}, \ldots, s_{n}\right)\right|^{2} d s_{1} \ldots d s_{n}<\infty
$$

then

$$
\left\|A_{I}^{+}(F) \psi\right\|^{2} \leq \int_{I} d t\left\|F_{t} \psi\right\|^{2}+\int_{I \times I} d s d t\left\|\left(F_{s} \psi\right)(t, .)\right\|^{2}
$$

where $\|\cdot\|$ is the norm defined by:

$$
\|\psi\|^{2}:=\sum_{n \geq 0} n\left\|\psi^{(n)}\right\|^{2}
$$

\section{Proof}

$$
\begin{aligned}
\left\langle A_{I}^{+}(F) \psi, A_{I}^{+}(F) \psi\right\rangle & =\int_{I} d s \int_{I} d t\left\langle a_{s}^{+} F_{s} \psi, a_{t}^{+} F_{t} \psi\right\rangle \\
& =\int_{I} d s \int_{I} d t\left\langle F_{s} \psi,\left[a_{s}, a_{t}^{+}\right] F_{t} \psi\right\rangle+\int_{I} d s \int_{I} d t\left\langle F_{s} \psi, a_{t}^{+} a_{s} F_{t} \psi\right\rangle \\
& =\int_{I} d t\left\langle F_{t} \psi, F_{t} \psi\right\rangle+\int_{I \times I} d s d t\left\langle a_{t} F_{s} \psi, a_{s} F_{t} \psi\right\rangle .
\end{aligned}
$$


Now consider the integral:

$$
\begin{aligned}
J & :=\int_{I} d s \int_{I} d t\left\langle a_{t} F_{s} \psi, a_{s} F_{t} \psi\right\rangle \\
& =\int_{I \times I} d s d t \sum_{n=0}^{\infty}(n+1) \int_{\mathbb{R}^{n d}} \overline{\left(F_{s} \psi\right)^{(n+1)}(t, \sigma)}\left(F_{t} \psi\right)^{(n+1)}(s, \sigma) d \sigma
\end{aligned}
$$

Using Cauchy Schwarts inequality and Lebesgue theorem, we get:

$$
\begin{aligned}
J \leq & \sum_{n=0}^{\infty} \frac{(n+1)}{2} \int_{I \times I} d s d t\left\|\left(F_{s} \psi\right)^{(n+1)}(t, .)\right\|_{L^{2}\left(\mathbb{R}^{n d}\right)}^{2} \\
& +\sum_{n=0}^{\infty} \frac{(n+1)}{2} \int_{I \times I} d s d t\left\|\left(F_{t} \psi\right)^{(n+1)}(s, .)\right\|_{L^{2}\left(\mathbb{R}^{n d}\right)}^{2} \\
= & \sum_{n=0}^{\infty}(n+1) \int_{I \times I} d s d t\left\|\left(F_{s} \psi\right)^{(n+1)}(t, .)\right\|_{L^{2}\left(\mathbb{R}^{n d}\right)}^{2} \\
= & \int_{I \times I} d s d t\left\|\left(F_{s} \psi\right)(t, .)\right\|^{2}
\end{aligned}
$$

where $\|$.$\| \| is the norm (3.4).$

Remark 3.1. This estimate in the multidimensional case is not useful to prove the convergence of iterated series, but as it follows, we can find a good estimate in the white noise adapted and onedimensional case.

\section{White NOISE ADAPTED STOCHASTIC INTEGRAL EQUATION}

In this section, we will show that the white noise adaptness condition gives the opportunity to have more precise results with more regularity. We will generalize some results proved by Hudson and Parthasarathy in two directions:

(i) the adaptness condition will be replaced by white noise adaptness [15]

(ii) the estimate will be valid not only on the exponential domain, but on the whole maximal algebraic domain.

At first, we recall that a stochastic process in $\mathcal{F}$, indexed by $\mathbb{R}_{+}$, is a family $\left(F_{t}\right)_{t \geq 0}$ of elements of $\mathcal{L}(D, \mathcal{F})$ satisfying that for each $\psi \in D$, the map $t \mapsto F_{t} \psi$ is Borel measurable. Alternatively, a stochastic process indexed by $\mathbb{R}_{+}$can be looked as a map $t \in \mathbb{R}_{+} \mapsto F_{t} \in \mathcal{L}(D, \mathcal{F})$ with the above mentioned measurability property. We remark that elements of $\mathcal{L}$ can be regarded as stochastic processes. In the next, we shall only deal with processes indexed by $I \subseteq \mathbb{R}_{+}$.

Definition 4.1. A process $\left(F_{t}\right)_{t \geq 0}$ is said to be white noise adapted if for any $m_{t} \in\left\{a_{t}, a_{t}^{+}, a_{t} a_{t}^{+}\right\}$, we have:

$$
\forall \psi \in \mathcal{D}_{M A D}, F_{s} m_{t} \psi=m_{t} F_{s} \psi, \forall s<t .
$$

Lemma 4.1. If a process $\left(F_{t}\right)_{t \geq 0}$ is adapted in the sense of Hudson and Parthasarathy, it is white noise adapted.

Proof. Let $\left(F_{t}\right)_{t \geq 0}$ an adapted process in the sense of Hudson and Parthasarathy [15], which means that for any exponential vector $\psi_{f}=\psi_{f_{t]}} \otimes \psi_{f_{[t}}$, for $t \in \mathbb{R}^{+}$one has:

$$
F_{t} \psi_{f}=\left(F_{t} \psi_{f_{t]}}\right) \otimes \psi_{f_{[t}}
$$


SO

$$
\begin{aligned}
a_{s} F_{t} \psi_{f} & =a_{s}\left(F_{t} \psi_{f_{t]}}\right) \otimes \psi_{f_{[t}}, \forall s>t \\
a_{s} F_{t} \psi_{f} & =\left(F_{t} \psi_{f_{t]}}\right) \otimes a_{s} \psi_{f_{[t}}, \forall s>t \\
& =F_{t} a_{s} \psi_{f}, \forall s>t
\end{aligned}
$$

Then, we conclude that $\left(F_{t}\right)_{t \geq 0}$ is white noise adapted.

4.1. The Hudson-Parthasarathy estimate. In the following section we will prove that the white noise approach allows to obtain a stronger result. In fact the next estimate was proved by Hudson and Parthasarathy [15], in the adapted case and here we given a different proof of the same result but for general kind of processes, and this using the white noise approach.

Proposition 4.1. For all strongly continuous processes $\left(F_{s}\right)_{s \geq 0}$ on $\mathcal{D}_{M A D}(C)$, we have:

$$
\left\|\int_{0}^{t} d s F_{s} a_{s} \psi_{f}\right\|^{2} \leq e^{t} \int_{0}^{t} d s\left\|F_{s} \psi_{f}\right\|^{2}|f(s)|^{2}
$$

Proof. Let $\psi_{f}$ be an exponential vector with test function $f$, then, we denote by:

SO

$$
I(t) \psi_{f}=\int_{0}^{t} d s F_{s} a_{s} \psi_{f}
$$

$$
\left\|I(t) \psi_{f}\right\|^{2}=\left\|\int_{0}^{t} d s F_{s} a_{s} \psi_{f}\right\|^{2}
$$

For fixed $t, d t>0$ and arbitrary function $F(t)$, we use the notation

$$
d F(t)=F(t+d t)-F(t)
$$

(finite difference). In this notation one has the algebraic identity:

$$
\begin{aligned}
d\left\langle I(t) \psi_{f}, I(t) \psi_{f}\right\rangle & =\left\langle d I(t) \psi_{f}, I(t) \psi_{f}\right\rangle+\left\langle d I(t) \psi_{f}, d I(t) \psi_{f}\right\rangle+\left\langle d I(t) \psi_{f}, d I(t) \psi_{f}\right\rangle \\
& =2 \operatorname{Re}\left\langle\int_{t}^{t+d t} d s F_{s} f(s) \psi_{f}, I(t) \psi_{f}\right\rangle+\left\langle\int_{t}^{t+d t} d t_{1} F_{t_{1}} f\left(t_{1}\right) \psi_{f}, \int_{t}^{t+d t} d t_{2} F_{t_{2}} f\left(t_{2}\right) \psi_{f}\right\rangle \\
& =2 \operatorname{Re} \int_{t}^{t+d t} d s \overline{f(s)}\left\langle F_{s} \psi_{f}, I(t) \psi_{f}\right\rangle+\int_{t}^{t+d t} d t_{1} \int_{t}^{t+d t} d t_{2} \overline{f\left(t_{1}\right)} f\left(t_{2}\right)\left\langle F_{t_{1}} \psi_{f}, F_{t_{2}} \psi_{f}\right\rangle .
\end{aligned}
$$

Denote

$$
h\left(t_{1}, t_{2}\right):=\overline{f\left(t_{1}\right)} f\left(t_{2}\right)\left\langle F_{t_{1}} \psi_{f}, F_{t_{2}} \psi_{f}\right\rangle
$$

Since the map $s \mapsto F_{s} \psi_{f}$ is continuous, we deduce that the map $\left(t_{1}, t_{2}\right) \mapsto h\left(t_{1}, t_{2}\right)$ is uniformly bounded on $[t, t+d t]$ for each $t \in \mathbb{R}_{+}$, in fact:

$$
\left|h\left(t_{1}, t_{2}\right)\right| \leq\|f\|_{\infty}^{2} \sup _{s \in[t, t+d t]}\left\|F_{s} \psi_{f}\right\|^{2}
$$

using this and the identity

$$
\begin{gathered}
\frac{1}{d t} d\left\langle I(t) \psi_{f}, I(t) \psi_{f}\right\rangle= \\
\frac{1}{d t} 2 \operatorname{Re}\left\langle\int_{t}^{t+d t} F_{s} f(s) d s \psi_{f}, I(t) \psi_{f}\right\rangle+\frac{1}{d t} \int_{t}^{t+d t} d t_{1} \int_{t}^{t+d t} d t_{2} h\left(t_{1}, t_{2}\right)
\end{gathered}
$$

we get, for $d t \rightarrow 0$$$
\frac{d}{d t}\left\langle I(t) \psi_{f}, I(t) \psi_{f}\right\rangle=2 \operatorname{Re}\left\langle F_{t} f(t) \psi_{f}, I(t) \psi_{f}\right\rangle
$$

therefore

$$
\frac{d}{d t}\left\langle I(t) \psi_{f}, I(t) \psi_{f}\right\rangle \leq\left\|F_{t} f(t) \psi_{f}\right\|^{2}+\left\|I(t) \psi_{f}\right\|^{2}
$$


Thus, by Gronwall lemma:

which is (4.2).

$$
\left\|I(t) \psi_{f}\right\|^{2} \leq e^{t} \int_{0}^{t} d t|f(t)|^{2}\left\|F_{t} \psi_{f}\right\|^{2}
$$

4.2. 1-dimensional, white noise adapted left creator estimate. We need the following preliminary result, which is true in arbitrary dimensions.

Lemma 4.2. For all $\psi$ in $\mathcal{D}_{M A D}(C)$ satisfying

$$
\|\psi\|^{2}:=\sum_{n \geq 0} n\left\|\psi^{(n)}\right\|^{2}<\infty .
$$

Then for each $s_{1} \in \mathbb{R}^{d}$, the following series:

$$
\sum_{n=0}^{\infty}(n+1) \int_{\mathbb{R}^{d n}} d s_{2} \cdots d s_{n+1}\left|\psi^{(n+1)}\left(s_{1}, s_{2}, \cdots, s_{n+1}\right)\right|^{2}
$$

is finite a.e.

\section{Proof}

Using the Dini theorem and (4.3), we get

$$
\|\psi\|^{2}=\int_{\mathbb{R}^{d}} d s_{1} \sum_{n=0}^{\infty}(n+1) \int_{\mathbb{R}^{d n}} d s_{2} \cdots d s_{n+1}\left|\psi^{(n+1)}\left(s_{1}, s_{2}, \cdots, s_{n+1}\right)\right|^{2}<\infty .
$$

Then, we deduce that for each $s_{1}$ in $\mathbb{R}^{d}$ we have

$$
\sum_{n=0}^{\infty}(n+1) \int_{\mathbb{R}^{d n}} d s_{2} \cdots d s_{n+1}\left|\psi^{(n+1)}\left(s_{1}, s_{2}, \cdots, s_{n+1}\right)\right|^{2}<\infty \text { a.e. }
$$

\section{Proposition 4.2. (1-dimensional left creator estimates)}

For all white noise adapted processes $\left(F_{s}\right)_{s \geq 0}$ and all $\psi \in \mathcal{D}_{M A D}(C)$ such that the map $\left(t_{1}, t_{2}\right) \mapsto$ $F_{t_{1}} \psi\left(t_{2},.\right)$ is continuous for $t_{1}, t_{2}$ in every interval of $\mathbb{R}_{+}$for the norm (3.4), we have, for any $0 \leq$ $t \leq T<+\infty$

$$
\left\|\int_{0}^{t} d s a_{s}^{+} F_{s} \psi\right\|^{2} \leq e^{\left(C_{T, \psi}|J(\psi)|\right) t} \sum_{\psi_{i} \in J(\psi)} \int_{0}^{t}\left\|F_{s} \psi_{i}\right\|^{2} d s
$$

where $J(\psi)$ is the subset of $\mathcal{F}$ defined by

$$
J(\psi)=\left\{\psi_{i}=A_{f_{n}}^{+} \ldots \hat{A}_{f_{i}}^{+} \ldots A_{f_{1}}^{+} \psi_{f}, A_{f_{n}}^{+} \ldots A_{f_{1}}^{+} \psi_{f} 1 \leq i \leq n\right\} \subset \mathcal{D}_{M A D},
$$

|.| denotes the cardinality,

$$
C_{T, \psi}=\max _{\psi_{i} \in J(\psi)} 2 c_{T, \psi_{i}}, c_{T, \psi_{i}}=\max _{1 \leq j \leq n}\left(\left\|f_{j}\right\|_{\infty, T}^{2},\|f\|_{\infty, T}^{2}\right) .
$$

where $\|f\|_{\infty, T}=\sup _{t \in[0, T]}|f(t)|$.

Proof. Let $\psi=A_{f_{n}}^{+} \cdots A_{f_{1}}^{+} \psi_{f}$, and

$$
A_{t}(F) \psi=\int_{0}^{t} d s a_{s}^{+} F_{s} \psi
$$

so to estimate $\left\|A_{t}(F) \psi\right\|^{2}$ it is sufficient to estimate $d\left\langle A_{t}(F) \psi, A_{t}(F) \psi\right\rangle$ for this we obtain

$$
d\left\langle A_{t}(F) \psi, A_{t}(F) \psi\right\rangle=\left\langle d A_{t}(F) \psi, A_{t}(F) \psi\right\rangle+\left\langle A_{t}(F) \psi, d A_{t}(F) \psi\right\rangle+\left\langle d A_{t}(F) \psi, d A_{t}(F) \psi\right\rangle
$$




$$
=2 \operatorname{Re}\left\langle d A_{t}(F) \psi, A_{t}(F) \psi\right\rangle+\left\langle d A_{t}(F) \psi, d A_{t}(F) \psi\right\rangle
$$

For the term $\left\langle d A_{t}(F) \psi, d A_{t}(F) \psi\right\rangle$, we obtain

$$
\begin{aligned}
\left\langle d A_{t}(F) \psi, d A_{t}(F) \psi\right\rangle= & \left\langle\int_{t}^{t+d t} d t_{1} a_{t_{1}}^{+} F_{t_{1}} \psi, \int_{t}^{t+d t} d t_{2} a_{t_{2}}^{+} F_{t_{2}} \psi\right\rangle \\
= & \int_{t}^{t+d t} d t_{1} \int_{t}^{t+d t} d t_{2}\left\langle a_{t_{1}}^{+} F_{t_{1}} \psi, a_{t_{2}}^{+} F_{t_{2}} \psi\right\rangle \\
= & \int_{t}^{t+d t} d t_{1} \int_{t}^{t+d t} d t_{2}\left\langle F_{t_{1}} \psi, a_{t_{1}} a_{t_{2}}^{+} F_{t_{2}} \psi\right\rangle \\
= & \int_{t}^{t+d t} d t_{1} \int_{t}^{t+d t} d t_{2}\left\langle F_{t_{1}} \psi, \delta\left(t_{1}-t_{2}\right) F_{t_{2}} \psi\right\rangle \\
& +\int_{t}^{t+d t} d t_{1} \int_{t}^{t+d t} d t_{2}\left\langle F_{t_{1}} \psi, a_{t_{2}}^{+} a_{t_{1}} F_{t_{2}} \psi\right\rangle \\
= & \int_{t}^{t+d t} d t_{1}\left\langle F_{t_{1}} \psi, F_{t_{1}} \psi\right\rangle \\
& +\int_{t}^{t+d t} d t_{1} \int_{t}^{t+d t} d t_{2}\left\langle a_{t_{2}} F_{t_{1}} \psi, a_{t_{1}} F_{t_{2}} \psi\right\rangle
\end{aligned}
$$

Denote by: $\mathcal{A}:=\int_{t}^{t+d t} d t_{1} \int_{t}^{t+d t} d t_{2}\left\langle a_{t_{2}} F_{t_{1}} \psi, a_{t_{1}} F_{t_{2}} \psi\right\rangle$ then, we get:

$$
\begin{aligned}
\mathcal{A}= & \int_{t}^{t+d t} d t_{1} \int_{t}^{t+d t} d t_{2} \sum_{n=0}^{\infty} \int_{\mathbb{R}^{n d}} \overline{\left(a_{t_{2}} F_{t_{1}} \psi\right)^{(n)}\left(s_{1}, s_{2}, \cdots, s_{n}\right)} \\
& \times\left(a_{t_{1}} F_{t_{2}} \psi\right)^{(n)}\left(s_{1}, s_{2}, \cdots, s_{n}\right) d s_{1} \ldots d s_{n} \\
= & \int_{t}^{t+d t} d t_{1} \int_{t}^{t+d t} d t_{2} \sum_{n=0}^{\infty}(n+1) \int_{\mathbb{R}^{n d}} \overline{\left(F_{t_{1}} \psi\right)^{(n+1)}\left(t_{2}, s_{1}, s_{2}, \cdots, s_{n}\right)} \\
& \times\left(F_{t_{2}} \psi\right)^{(n+1)}\left(t_{1}, s_{1}, s_{2}, \cdots, s_{n}\right) d s_{1} \ldots d s_{n} \\
= & \int_{t}^{t+d t} d t_{1} \int_{t}^{t+d t} d t_{2} g\left(t_{1}, t_{2}\right)
\end{aligned}
$$

where:

$$
\begin{aligned}
g\left(t_{1}, t_{2}\right) & :=\sum_{n=0}^{\infty}(n+1) \int_{\mathbb{R}^{d n}} \overline{\left(F_{t_{1}} \psi\right)^{(n+1)}\left(t_{2}, \sigma\right)}\left(F_{t_{2}} \psi\right)^{(n+1)}\left(t_{1}, \sigma\right) d \sigma \text { a.e. } \\
& =:\left\langle N F_{t_{1}} \psi\left(t_{2}, .\right), F_{t_{2}} \psi\left(t_{1}, .\right)\right\rangle
\end{aligned}
$$

The continuity of the map: $\left(t_{1}, t_{2}\right) \mapsto g\left(t_{1}, t_{2}\right)$ a.e. on $[t, t+d t]^{2}$, is a consequence of the following inequalities: $\forall \epsilon>0$

$$
\begin{aligned}
g\left(t_{1}+\epsilon, t_{2}+\epsilon\right)-g\left(t_{1}, t_{2}\right)= & \left\langle N F_{t_{1}+\epsilon} \psi\left(t_{2}+\epsilon, .\right), F_{t_{2}+\epsilon} \psi\left(t_{1}+\epsilon, .\right)\right\rangle-\left\langle N F_{t_{1}} \psi\left(t_{2}, .\right), F_{t_{2}} \psi\left(t_{1}, .\right)\right\rangle \\
= & \left\langle N\left(F_{t_{1}+\epsilon} \psi\left(t_{2}+\epsilon, .\right)-F_{t_{1}} \psi\left(t_{2}, .\right)\right), F_{t_{2}+\epsilon} \psi\left(t_{1}+\epsilon, .\right)\right\rangle \\
& +\left\langle N F_{t_{1}} \psi\left(t_{2}, .\right), F_{t_{2}+\epsilon} \psi\left(t_{1}+\epsilon, .\right)-F_{t_{2}} \psi\left(t_{1}, .\right)\right\rangle \\
\leq & \| \sqrt{N}\left(F_{t_{1}+\epsilon} \psi\left(t_{2}+\epsilon, .\right)-F_{t_{1}} \psi\left(t_{2}, .\right)\|.\| F_{t_{2}+\epsilon} \psi\left(t_{1}+\epsilon, .\right) \|\right. \\
& +\left\|\sqrt{N} F_{t_{1}} \psi\left(t_{2}, .\right)\right\| . \|\left(F_{t_{2}+\epsilon} \psi\left(t_{1}+\epsilon, .\right)-F_{t_{2}} \psi\left(t_{1}, .\right) \|\right.
\end{aligned}
$$

and of the continuity of $\left(t_{1}, t_{2}\right) \mapsto F_{t_{1}} \psi\left(t_{2},.\right)$ in the norm (3.4). Since g is bounded in $[t, t+d t]^{2}$ it follows that: $|\mathcal{A}| \leq \beta(d t)^{2}$, where $\beta>0$, i.e. $\mathcal{A}=O\left(d t^{2}\right)$. In conclusion:

$$
\frac{1}{d t} d\left\langle A_{t}(F) \psi, A_{t}(F) \psi\right\rangle=\frac{1}{d t} 2 \operatorname{Re}\left\langle\int_{t}^{t+d t} d s a_{s}^{+} F_{s} \psi, A_{t}(F) \psi\right\rangle+\frac{1}{d t} \int_{t}^{t+d t} d t_{1}\left\langle F_{t_{1}} \psi, F_{t_{1}} \psi\right\rangle+\frac{1}{d t} \mathcal{A}
$$


and, if $d t \rightarrow 0$, we get

$$
\frac{d}{d t}\left\langle A_{t}(F) \psi, A_{t}(F) \psi\right\rangle=2 \operatorname{Re}\left\langle a_{t}^{+} F_{t} \psi, \int_{0}^{t} d s a_{s}^{+} F_{s} \psi\right\rangle+\left\langle F_{t} \psi, F_{t} \psi\right\rangle
$$

Let $\psi=A_{f_{m}}^{+} \ldots A_{f_{1}}^{+} \psi_{f}$ in $\mathcal{D}_{M A D}(C)$ where $f, f_{1} \ldots f_{m}$ are test functions in $C$, and $F_{t}$ is a white noise adapted process, for each $t$, so we obtain:

$$
\begin{aligned}
\left\langle a_{t}^{+} F_{t} \psi, \int_{0}^{t} d s a_{s}^{+} F_{s} \psi\right\rangle & =\int_{0}^{t} d s\left\langle F_{t} \psi, a_{t} a_{s}^{+} F_{s} \psi\right\rangle \\
& =\int_{0}^{t} d s\left\langle F_{t} \psi, a_{s}^{+} F_{s} a_{t} \psi\right\rangle \\
& =\int_{0}^{t} d s\left\langle F_{t} \psi, a_{s}^{+} F_{s} a_{t} \psi\right\rangle
\end{aligned}
$$

since:

$$
\begin{aligned}
\int_{0}^{t} d s a_{s}^{+} F_{s} a_{t} A_{f_{n}}^{+} \ldots A_{f_{1}}^{+} \psi_{f} & =\int_{0}^{t} d s a_{s}^{+} F_{s}\left[a_{t}, A_{f_{n}}^{+} \ldots A_{f_{1}}^{+}\right] \psi_{f} d s+\int_{0}^{t} d s a_{s}^{+} F_{s} d s f(t) A_{f_{n}}^{+} \ldots A_{f_{1}}^{+} \psi_{f} \\
& =\int_{0}^{t} d s a_{s}^{+} F_{s}\left[\sum_{i=1}^{n} f_{i}(t) A_{f_{n}}^{+} \ldots \hat{A}_{f_{i}}^{+} \ldots A_{f_{1}}^{+} \psi_{f}+f(t) A_{f_{n}}^{+} \ldots A_{f_{1}}^{+} \psi_{f}\right]
\end{aligned}
$$

so:

$$
\begin{gathered}
\left\|\int_{0}^{t} d s a_{s}^{+} F_{s} a_{s} A_{f_{n}}^{+} \ldots \hat{A}_{f_{i}}^{+} \ldots A_{f_{i}}^{+} \psi_{f}\right\|^{2} \\
=\left\|\int_{0}^{t} d s a_{s}^{+} F_{s}\left[\sum_{i=1}^{n} f_{i}(t) A_{f_{n}}^{+} \ldots \hat{A}_{f_{i}}^{+} \ldots A_{f_{1}}^{+} \psi_{f}+f(t) A_{f_{n}}^{+} \ldots A_{f_{1}}^{+} \psi_{f}\right]\right\|^{2} \\
=\left\|\sum_{i=1}^{n}\left[\int_{0}^{t} d s a_{s}^{+} F_{s} f_{i}(t) A_{f_{n}}^{+} \ldots \hat{A}_{f_{i}}^{+} \ldots A_{f_{i}}^{+} \psi_{f}\right]+\int_{0}^{t} d s a_{s}^{+} F_{s} f(t) A^{+} f_{n} \ldots A_{f_{1}}^{+} \psi_{f}\right\|^{2} \\
\leq 2\left\|\sum_{i=1}^{n} \int_{0}^{t} d s f_{i}(t) a_{s}^{+} F_{s} A_{f_{n}}^{+} \ldots \hat{A}_{f_{i}}^{+} \ldots A_{f_{1}}^{+} \psi_{f}\right\|^{2}+2\left\|\int_{0}^{t} d s a_{s}^{+} F_{s} f(t) A_{f_{n}}^{+} \ldots A_{f_{1}}^{+} \psi_{f}\right\|^{2} \\
\leq 2 c_{T, \psi}\left[\sum_{i=1}^{n}\left\|\int_{0}^{t} d s a_{s}^{+} F_{s} A_{f_{n}}^{+} \ldots \hat{A}_{f_{i}}^{+} \ldots A_{f_{1}}^{+} \psi_{f}\right\|^{2}+\left\|\int_{0}^{t} d s a_{s}^{+} F_{s} A_{f_{n}}^{+} \ldots A_{f_{1}}^{+} \psi_{f}\right\|^{2}\right],
\end{gathered}
$$

where

$$
c_{T, \psi}=\max _{1 \leq i \leq n}\left(\left\|f_{i}\right\|_{\infty, T}^{2},\|f\|_{\infty, T}^{2}\right)
$$

then

$$
\begin{gathered}
\frac{d}{d t}\left\langle A_{t}(F) \psi, A_{t}(F) \psi\right\rangle \leq \\
2\left\|F_{t} \psi\right\|^{2}+2 c_{T, \psi}\left[\left\|A_{t}(F) \psi\right\|^{2}+\sum_{i=1}^{n}\left\|\int_{0}^{t} d s a_{s}^{+} F_{s} A_{f_{n}}^{+} \ldots \hat{A}_{f_{i}}^{+} \ldots A_{f_{1}}^{+} \psi_{f}\right\|^{2}\right]
\end{gathered}
$$

We denote by

$$
\left\|A_{t}(F) \psi\right\|_{F_{\psi}}^{2}=\sum_{\psi_{i} \in F_{\psi}}\left\|A_{t}(F) \psi_{i}\right\|^{2}
$$

where

$$
F_{\psi}=\left\{\psi_{i}=A_{f_{n}}^{+} \ldots \hat{A}_{f_{i}}^{+} \ldots A_{f_{1}}^{+} \psi_{f}, A_{f_{n}}^{+} \ldots A_{f_{1}}^{+} \psi_{f}, 1 \leq i \leq n\right\}
$$

We note that $\forall \psi_{i} \in F_{\psi}$ :

$$
\left\|A_{t}(F) \psi_{i}\right\|^{2} \leq\left\|A_{t}(F) \psi\right\|_{F_{\psi}}^{2}
$$


and

$$
\begin{gathered}
\left\|A_{t}(F) \psi_{i}\right\|^{2} \leq \int_{0}^{t} d s\left\|F_{s} \psi_{i}\right\|^{2}+2 c_{T, \psi_{i}} \sum_{j} \int_{0}^{t} d s\left\|A_{s}(F) \psi_{i j}\right\|^{2} \\
\left\|A_{t}(F) \psi\right\|_{F_{\psi}}^{2} \leq \int_{0}^{t} \sum_{\psi_{i} \in F_{\psi}}\left\|F_{s} \psi_{i}\right\|^{2} d s+C_{T, \psi}\left|F_{\psi}\right| \int_{0}^{t}\left\|A_{s}(F) \psi\right\|_{F_{\psi}}^{2} d s
\end{gathered}
$$

where $C_{T, \psi}=\max _{\psi_{i} \in F} 2 c_{T, \psi_{i}}$.

$$
\left\|A_{t}(F) \psi\right\|_{F_{\psi}}^{2} \leq \sum_{\psi_{i} \in F_{\psi}} \int_{0}^{t}\left\|F_{s} \psi_{i}\right\|^{2} d s+C_{T, \psi}\left|F_{\psi}\right| \int_{0}^{t}\left\|A_{s}(F) \psi\right\|_{F_{\psi}}^{2} d s
$$

by Gronwall lemma, we obtain

$$
\left\|A_{t}(F) \psi\right\|^{2} \leq\left\|A_{t}(F) \psi\right\|_{F_{\psi}}^{2} \leq e^{\left(C_{T, \psi}\left|F_{\psi}\right|\right) t} \sum_{\psi_{i} \in F_{\psi}} \int_{0}^{t}\left\|F_{s} \psi_{i}\right\|^{2} d s .
$$

So we get the result where $J(\psi)=F_{\psi}$.

Corollary 4.1. For $\psi=\psi_{f}$, we have, using the fact that $F_{t}$ is white noise adapted and Gromwall lemma:

$$
\left\|\int_{0}^{t} d s a_{s}^{+} F_{s} \psi_{f}\right\|^{2} \leq e^{\|f\|_{\infty, T} t} \int_{0}^{t} d s\left[\|f\|_{\infty}+1\right]\left\|F_{s} \psi_{f}\right\|^{2} .
$$

The above estimate is similar to those proved by Hudson and Parthasaraty on the exponential vectors.

Corollary 4.2. Let $\left(F_{t}\right)_{t \geq 0}$ be a white noise adapted process such that, for each $n \in \mathbb{N}^{*}$ and test functions $f, f_{1} \cdots f_{n}$ in $C$, the map

$$
\left(t_{1}, t_{2}\right) \mapsto F_{t_{1}} \psi\left(t_{2}, .\right) ; \quad \psi=A_{f_{n}}^{+} \cdots A_{f_{1}}^{+} \psi_{f} \in \mathcal{D}_{M A D}(C)
$$

is continuous under the norm (3.4). Then the following inequality holds for any $0 \leq t \leq T<\infty$ :

$$
\left\|\int_{0}^{t} d s a_{s}^{+} F_{s} a_{s} \psi\right\|^{2} \leq c_{T, \psi}^{\prime} \int_{0}^{t} d s \sum_{\psi_{i} \in J(\psi)}\left\|F_{s} \psi_{i}\right\|^{2}
$$

where $J(\psi) \subset \mathcal{D}, c_{T, \psi}^{\prime}$ are defined by

$$
\begin{aligned}
& J(\psi)=\left\{A_{f_{n}}^{+} \cdots A_{f_{1}}^{+} \psi_{f}, A_{f_{n}}^{+} \cdots \hat{A}_{f_{i}}^{+} \cdots A_{f_{1}}^{+} \psi_{f},: 1 \leq i \leq n\right\} \\
& c_{T, \psi}^{\prime}=2 n \max _{1 \leq i \leq n}\left(\left\|f_{i}\right\|_{\infty, T}^{2},\|f\|_{\infty, T}^{2}\right) c_{T, \psi} \max _{1 \leq i \leq n}\left(e^{\left(c_{T, \psi}|F|\right) T}, e^{\left(c_{T, \psi_{i}}\left|F_{i}\right|\right) T}\right)
\end{aligned}
$$

where $\left\|f_{i}\right\|_{\infty, T}=\sup _{s \in[0, T]}|f(s)|$ and $c_{T, \psi}, c_{T, \psi_{i}}$ are defined as in (4.1.a) 
Proof. Using lemma (4.1), we get:

$$
\begin{aligned}
& \left\|\int_{0}^{t} d s a_{s}^{+} F_{s} a_{s} \psi\right\|^{2}=\left\|\int_{0}^{t} d s a_{s}^{+} F_{s}\left[\sum_{i=1}^{n} F_{s} f_{i}(s) A_{f_{n}}^{+} \cdots \hat{A}_{f_{i}}^{+} \cdots A_{f_{1}}^{+} \psi_{f}+f(s) A_{f_{n}}^{+} \cdots A_{f_{1}}^{+} \psi_{f}\right]\right\|^{2} \\
& \leq 2\left\|\int_{0}^{t} d s a_{s}^{+} F_{s} \sum_{i=1}^{n} F_{s} f_{i}(s) A_{f_{n}}^{+} \cdots \hat{A}_{f_{i}}^{+} \cdots A_{f_{1}}^{+} \psi_{f}\right\|^{2} \\
& +2\left\|\int_{0}^{t} d s a_{s}^{+} F_{s} f(s) A_{f_{n}}^{+} \cdots A+_{f_{1}} \psi_{f}\right\|^{2} \\
& \leq 2 n \cdot \max _{1 \leq i \leq n}\left(\left\|f_{i}\right\|_{\infty, T}^{2}\right)\left[\sum_{i=1}^{n}\left\|\int_{0}^{t} d s a_{s}^{+} F_{s} A_{f_{n}}^{+} \cdots \hat{A_{f_{i}}^{+}} \cdots A_{f_{1}}^{+} \psi\right\|^{2}\right] \\
& +2\|f\|_{\infty, T}^{2}\left\|\int_{0}^{t} d s a_{s}^{+} F_{s} A_{f_{n}}^{+} \cdots A+_{f_{1}} \psi_{f}\right\|^{2} \\
& \leq 2 \max _{1 \leq i \leq n}\left(n\left\|f_{i}\right\|_{\infty, T}^{2}\|f\|_{\infty, T}^{2}\right) \max _{1 \leq i \leq n}\left(e^{\left(c_{T, \psi}|F|\right) T}, e^{\left(c_{T, \psi_{i}}\left|F_{i}\right|\right) T}\right) \\
& \times \sum_{i=1}^{n}\left[\int_{0}^{t} d s\left\|F_{s} A_{f_{n}}^{+} \cdots \hat{A}_{f_{i}}^{+} \cdots A_{f_{1}}^{+} \psi_{f}\right\|^{2}\right]+\int_{0}^{t} d s\left\|F_{s} A_{f_{n}}^{+} \cdots A+_{f_{1}} \psi_{f}\right\|^{2} .
\end{aligned}
$$

where $\left(c_{T, \psi}|F|\right),\left(c_{T, \psi_{i}}\left|F_{i}\right|\right)$ are defined as in (4.1.a).

Corollary 4.3. Let $\left(F_{t}\right)_{t \geq 0}$ be a white noise adapted process such that, for each $n \in \mathbb{N}^{*}$ and test functions $f, f_{1} \cdots f_{n}$ in $C$, the map

$$
\left(t_{1}, t_{2}\right) \mapsto F_{t_{1}} \psi\left(t_{2}, .\right) ; \quad \psi=A_{f_{n}}^{+} \cdots A_{f_{1}}^{+} \psi_{f} \in \mathcal{D}_{M A D}(C)
$$

is continuous under the norm (3.4). Then the following inequalities hold for any $0 \leq u \leq t \leq T<\infty$ :

a-:

$$
\left\|\int_{u}^{t} d s F_{s} a_{s} \psi\right\| \leq c_{\psi, I} \sum_{\varphi \in J(\psi)}\left(\int_{u}^{t} d s\left\|F_{s} \varphi\right\|^{2}\right)^{\frac{1}{2}}
$$

where

and

$$
J(\psi)=\left\{A_{f_{n}}^{+} \ldots \hat{A}_{f_{i}}^{+} \ldots A_{f_{1}}^{+} \psi_{f}, A_{f_{n}}^{+} \ldots A_{f_{1}}^{+} \psi_{f}, 1 \leq i \leq n\right\}
$$

$$
c_{\psi, I}=\max _{1 \leq i \leq n}\left(\left\|f_{i}\right\|_{2, I},\|f\|_{2, I}\right)
$$

b-:

$$
\left\|\int_{u}^{t} d s a_{s}^{+} F_{s} \psi\right\|^{2} \leq e^{\left(C_{T, \psi}|J(\psi)|\right) t} \sum_{\psi_{i} \in J(\psi)} \int_{u}^{t}\left\|F_{s} \psi_{i}\right\|^{2} d s
$$

where $J(\psi)$ is the subset of $\mathcal{F}$ defined by

$$
J(\psi)=\left\{\psi_{i}=A_{f_{n}}^{+} \ldots \hat{A}_{f_{i}}^{+} \ldots A_{f_{1}}^{+} \psi_{f}, A_{f_{n}}^{+} \ldots A_{f_{1}}^{+} \psi_{f} 1 \leq i \leq n\right\} \subset \mathcal{D}_{M A D},
$$

|.| denotes the cardinality,

$$
C_{T, \psi}=\max _{\psi_{i} \in J(\psi)} 2 c_{T, \psi_{i}}, c_{T, \psi_{i}}=\max _{1 \leq j \leq n}\left(\left\|f_{j}\right\|_{\infty, T}^{2},\|f\|_{\infty, T}^{2}\right) .
$$

where $\|f\|_{\infty, T}=\sup _{t \in[0, T]}|f(t)|$.

$$
\left\|\int_{u}^{t} d s a_{s}^{+} F_{s} a_{s} \psi\right\|^{2} \leq c_{T, \psi}^{\prime} \int_{u}^{t} d s \sum_{\psi_{i} \in J(\psi)}\left\|F_{s} \psi_{i}\right\|^{2}
$$


where $J(\psi) \subset \mathcal{D}, c_{T, \psi}^{\prime}$ are defined by

$$
\begin{gathered}
J(\psi)=\left\{A_{f_{n}}^{+} \cdots A_{f_{1}}^{+} \psi_{f}, A_{f_{n}}^{+} \cdots \hat{A}_{f_{i}}^{+} \cdots A_{f_{1}}^{+} \psi_{f},: 1 \leq i \leq n\right\} \\
c_{T, \psi}^{\prime}=2 n \max _{1 \leq i \leq n}\left(\left\|f_{i}\right\|_{\infty, T}^{2},\|f\|_{\infty, T}^{2}\right) c_{T, \psi} \max _{1 \leq i \leq n}\left(e^{\left(c_{T, \psi}|F|\right) T}, e^{\left(c_{T, \psi_{i}}\left|F_{i}\right|\right) T}\right) \\
\text { where }\left\|f_{i}\right\|_{\infty, T}=\sup _{s \in[0, T]}|f(s)| \text { and } c_{T, \psi}, c_{T, \psi_{i}} \text { are defined as in (4.1.a). }
\end{gathered}
$$

Proof. a- is obtained by taking $I=[u, t]$ in proposition 3.1. Also the proof of b- and c- are obtained by replacing $o$ by $u$ in those of proposition 4.2 and corollary 4.1 .

Corollary 4.4. Let $\mathcal{H}_{S}$ a Hilbert space called initial space. The inequalities (3.3), (4.4), (4.5) are verified if we take

$$
\psi=u \otimes \psi \in \mathcal{H}_{S} \otimes \mathcal{F}
$$

where $u$ is in the initial space $\mathcal{H}_{S}$ and $\psi$ is an element of the maximal algebraic domain.

Proof. Using the fact that:

$$
F_{t} u \otimes \psi=u \otimes F_{t} \psi
$$

and the inequalities (3.3), (4.4) and (4.5), one has:

$$
\begin{gathered}
\left\|\int_{I} F_{s} a_{s} d s u \otimes \psi\right\| \leq c_{\psi, I} \sum_{\varphi \in J(\psi)}\left(\int_{I} d s\left\|u \otimes F_{s} \varphi\right\|^{2}\right)^{\frac{1}{2}} \\
\left\|\int_{0}^{t} d s a_{s}^{+} F_{s} u \otimes \psi\right\|^{2} \leq e^{\left(c_{T, \psi}|J(\psi)|\right) t} \sum_{\psi_{i} \in J(\psi)} \int_{0}^{t}\left\|u \otimes F_{s} \psi_{i}\right\|^{2} d s \\
\left\|\int_{0}^{t} a_{s}^{+} F_{s} a_{s} d s u \otimes \psi\right\|^{2} \leq c_{T, \psi}^{\prime} \int_{0}^{t} d s \sum_{\psi_{i} \in J(\psi)}\left\|u \otimes F_{s} \psi_{i}\right\|^{2}
\end{gathered}
$$

where $c_{\psi, I}, c_{T, \psi}$ and $c_{T, \psi}^{\prime}$ are defined in propositions (3.1), (4.2) and Corollary (4.1).

\section{Normally ORDERED White NOISE STOCHASTIC DIFFERENTIAL EQUATION}

5.1. Introduction and definitions. The normally ordered white noise stochastic differential equation:

$$
\partial_{t} U_{t}=a_{t}^{+} E_{t} U_{t}+F_{t} U_{t} a_{t}+a_{t}^{+} G_{t} U_{t} a_{t}+H_{t} U_{t} \quad U(0)=U_{0}
$$

where $\left(E_{t}\right)_{t \geq 0},\left(F_{t}\right)_{t \geq 0},\left(G_{t}\right)_{t \geq 0}$ and $\left(H_{t}\right)_{t \geq 0}$ be measurable and locally bounded operators acting on the initial space $\mathcal{H}_{S}$.

The meaning of equation (5.1) can be specified in two different ways:

(i): as an integral equation, i.e.

$$
U_{t}=U_{0}+\int_{0}^{t} d s a_{s}^{+} E_{s} U_{s}+\int_{0}^{t} d s F_{s} U_{s} a_{s}+\int_{0}^{t} d s a_{s}^{+} G_{s} U_{s} a_{s}+\int_{0}^{t} d s H_{s} U_{s}
$$

where the integrals on the right hand side are defined in section (3.1).

(ii): as a weak equation in some domain $D^{1}$ ( our choice of this domain will be specified later):

$$
\partial_{t}\left\langle\phi, U_{t} \psi\right\rangle=\left\langle a_{t} \phi, E_{t} U_{t} \psi\right\rangle+\left\langle\phi, F_{t} U_{t} a_{t} \psi\right\rangle+\left\langle a_{t} \phi, E_{t} U_{t} a_{t} \psi\right\rangle+\left\langle\phi, E_{t} U_{t} \psi\right\rangle
$$

where $\phi$ and $\psi$ are in $D^{1}$.

Since also the notion of stochastic integral requires the specification of some domain, the two methods may lead to inequivalent notions of solution. However the main goal of the theory is to produce solutions which are unitary (in particular bounded). Within this class it can be proved that, for a large family of coefficient processes (including the constant bounded ones, which are the most used in applications) the two equations lead to the same solution. 
5.2. White noise adapted normally ordered white noise equation in $\mathbb{R}^{d}$. Using the above results, we will prove the existence and the uniqueness of solution of a class of whit noise adapted normally ordered white noise equation by:

Definition 5.1. A white noise adapted normally ordered white noise equation with coefficients is a normally ordered white noise equation as defined above:

$$
\partial_{t} U_{t}=\left[F_{t}^{1} U_{t} a_{t}+a_{t}^{+} F_{t}^{2} U_{t}+a_{t}^{+} F_{t}^{3} U_{t} a_{t}+F_{t}^{4} U_{t}\right] d t
$$

where $\left(F_{t}^{1}\right)_{t \geq 0},\left(F_{t}^{2}\right)_{t \geq 0},\left(F_{t}^{3}\right)_{t \geq 0}$ and $\left(F_{t}^{4}\right)_{t \geq 0}$ are, white noise adapted processes in $B\left(\mathcal{H}_{S}\right)$ continuous for the norm operator topology on $\mathcal{B}\left(\mathcal{H}_{S}\right)$.

\subsection{Existence and uniqueness of the solution of a WN stocahstic equation.}

Theorem 5.1. Consider the normally ordered white noise equation:

$$
U_{t}=U_{0}+\int_{0}^{t} d s F_{s}^{1} U_{s} a_{s}+\int_{0}^{t} d s a_{s}^{+} F_{s}^{2} U_{s}+\int_{0}^{t} d s a_{s}^{+} F_{s}^{3} U_{s} a_{s}+\int_{0}^{t} d s F_{s}^{4} U_{s}
$$

where $\left(F_{s}^{1}\right)_{s \geq 0},\left(F_{s}^{2}\right)_{s \geq 0},\left(F_{s}^{3}\right)_{s \geq 0}$ and $\left(F_{s}^{4}\right)_{s \geq 0}$ are locally bounded continuous processes for the norm operator topology on $\mathcal{B}\left(\mathcal{H}_{S}\right)$ such that the maps:

$$
\left(t_{1}, t_{2}\right) \mapsto F_{t_{1}}^{2} \psi\left(t_{2}, .\right),\left(t_{1}, t_{2}\right) \mapsto F_{t_{1}}^{3} \psi\left(t_{2}, .\right)
$$

are continuous for the norm (3.4) for each $\psi \in \mathcal{D}_{M A D}(C)$ and $t_{1}, t_{2} \in \mathbb{R}_{+}^{*}$. Under the above conditions, equation (5.5) has a unique white noise adapted locally bounded continuous solution for all $U_{0}$ white noise adapted strongly continuous process on $\mathcal{D}_{M A D}(C)$.

Proof. i) Existence. Define by induction $U_{t}^{0}=U^{0}$, and

$$
U_{t}^{n+1}=\int_{0}^{t} F_{s}^{1} U_{s}^{n} a_{s} d s+\int_{0}^{t} a_{s}^{+} F_{s}^{2} U_{s}^{n} d s+\int_{0}^{t} a_{s}^{+} F_{s}^{3} U_{s}^{n} a_{s} d s+\int_{0}^{t} F_{s}^{4} U_{s}^{n} d s
$$

For simplicity, we use the notation:

$$
U_{t}^{n+1}=\int_{0}^{t} F_{s}^{i} U_{s}^{n} d M_{s}^{i}
$$

The sequence $\left(U_{t}^{n}\right)_{n \in \mathbb{N}}$ is well defined. In fact $U^{0}$ is a strongly continuous process on $\mathcal{D}_{M A D}(C)$. Suppose that $\forall 0 \leq k \leq n, U_{t}^{k}$ is a white noise adapted process on $\mathcal{D}_{M A D}(C)$, strongly continuous and such that the map :

$$
\left(t_{1}, t_{2}\right) \mapsto U_{t_{1}}^{k} \psi\left(t_{2}, .\right)
$$

is continuous for the norm (3.4). Using the scalar type integrator inequalities (3.3), (4.4) and (4.5), we have for any $0 \leq u \leq t \leq T$, and each $i \in\{1,2,3,4\}$ :

$$
\begin{gathered}
\left\|\int_{u}^{t} F_{s}^{1} U_{s}^{n} a_{s} d s \xi\right\|^{2} \leq\left|J_{1}(\xi)\right|\left(c_{T, 1, \eta}\right)^{2} \sum_{\eta \in J_{1}(\xi)} \int_{u}^{t} d s\left\|F_{s}^{1} U_{s}^{n} \eta\right\|^{2} \\
\left\|\int_{u}^{t} a_{s}^{+} F_{s}^{2} U_{s}^{n} d s\right\|^{2} \leq c_{T, 2, \eta} \sum_{\eta \in J_{2}(\xi)} \int_{u}^{t} d s\left\|F_{s}^{2} U_{s}^{n} \eta\right\|^{2} \\
\left\|\int_{u}^{t} a_{s}^{+} F_{s}^{3} U_{s}^{n} a_{s} d s\right\|^{2} \leq c_{T, 3, \eta} \sum_{\eta \in J_{3}(\xi)} \int_{u}^{t} d s\left\|F_{s}^{3} U_{s}^{n} \eta\right\|^{2} \\
\left\|\int_{u}^{t} F_{s}^{4} U_{s}^{n} d s\right\|^{2} \leq c_{T, 4, \eta} \sum_{\eta \in J_{4}(\xi)} \int_{u}^{t} d s\left\|F_{s}^{4} U_{s}^{n} \eta\right\|^{2}
\end{gathered}
$$


where $c_{T, i, \eta}, J_{i}(\xi), i=1,2,3,4$ are defined as in (3.3), (4.4)and (4.5). Then

$$
\begin{aligned}
\left\|U^{n}(t) \xi\right\|^{2} & =\left\|\int_{u}^{t} d M_{t_{1}}^{i} F^{i}\left(t_{1}\right) U_{t_{1}}^{n-1} \xi\right\|^{2} \\
& \leq c_{T, \xi} K_{T} \sum_{\xi_{1} \in J(\xi)} \int_{u}^{t} d t_{1}\left\|U_{t_{1}}^{n-1} \xi_{1}\right\|^{2}
\end{aligned}
$$

where:

$$
c_{T, \xi}=4 \max _{i \in\{1,2,3,4\}, \eta \in J(\xi)} c_{T, i, \eta} ; J(\xi)=\bigcup_{i} J_{i}(\xi), K_{T}=\sup _{s \in[0, T]}\left\|F_{s}\right\|_{\infty} .
$$

Let us now prove that $U^{n+1}$ satisfy (10.10). In fact $\forall \epsilon, \epsilon^{\prime}>0$ :

$$
\begin{aligned}
\left\|U_{t_{1}+\epsilon}^{n+1} \psi\left(t_{2}+\epsilon^{\prime}, .\right)-U_{t_{1}}^{n+1} \psi\left(t_{2}, .\right)\right\| \leqslant & \left\|U_{t_{1}+\epsilon}^{n+1} \psi\left(t_{2}+\epsilon^{\prime}, .\right)-U_{t_{1}}^{n+1} \psi\left(t_{2}+\epsilon^{\prime}, .\right)\right\| \\
& +\left\|U_{t_{1}}^{n+1} \psi\left(t_{2}+\epsilon^{\prime}, .\right)-U_{t_{1}}^{n+1} \psi\left(t_{2}, .\right)\right\| \\
= & \left\|\int_{t_{1}}^{t_{1}+\epsilon} d s F_{s}^{i} U_{s}^{n} d M_{s}^{i} \psi\left(t_{2}+\epsilon^{\prime}, .\right)\right\| \\
& +\left\|\int_{0}^{t_{1}} d s F_{s}^{i} U_{s}^{n} d M_{s}^{i} \psi\left(t_{2}+\epsilon^{\prime}, .\right)-\psi\left(t_{2}, .\right)\right\|
\end{aligned}
$$

using the inequality 5.6, we get:

$$
\begin{array}{r}
\left\|U_{t_{1}+\epsilon}^{n+1} \psi\left(t_{2}+\epsilon^{\prime}, .\right)-U_{t_{1}}^{n+1} \psi\left(t_{2}, .\right)\right\| \\
\left\{c_{t_{1}, \psi} \sum_{\psi_{i}} \int_{t_{1}}^{t_{1}+\epsilon} d s\left\|F_{s}^{i} U_{s}^{n} \psi_{i}\left(t_{2}+\epsilon^{\prime}, .\right)\right\|^{2}\right\}^{\frac{1}{2}}+\left\{c_{t_{1}, \psi}^{1} \sum_{\psi_{j}} \int_{0}^{t_{1}} d s \| F_{s}^{i} U_{s}^{n}\left[\psi_{j}\left(t_{2}+\epsilon^{\prime}, .\right)-\psi\left(t_{2}, .\right) \|^{2}\right\}^{\frac{1}{2}}\right.
\end{array}
$$

using our hypothesis, we have the continuity of the map

$$
s \mapsto\left\|F_{s}^{i} U_{s}^{n} \psi_{i}\left(t_{2}+\epsilon^{\prime}, .\right)\right\|
$$

so it will be uniform bounded on the compact $[t, t+\epsilon]$, then, we obtain that:

$$
\left\{c_{t_{1}, \psi} \sum_{\psi_{i}} \int_{t_{1}}^{t_{1}+\epsilon} d s\left\|U_{s}^{n} \psi_{i}\left(t_{2}+\epsilon^{\prime}, .\right)\right\|^{2}\right\}^{\frac{1}{2}}<K \epsilon, K>0
$$

Moreover under the assumption, the terms

$$
\left\{c_{t_{1}, \psi}^{1} \sum_{\psi_{j}} \int_{0}^{t_{1}} d s \| U_{s}^{n}\left[\psi_{j}\left(t_{2}+\epsilon^{\prime}, .\right)-\psi\left(t_{2}, .\right) \|^{2}\right\}^{\frac{1}{2}}\right.
$$

vanish when $\epsilon^{\prime} \rightarrow 0$,so we get the result.

Because of the above hypothesis of the induction, for all $M_{s}^{i} \in\left\{a_{s}, a_{s}^{+}, a_{s} a_{s}^{+}\right\}$and $i \in\{1,2,3,4\}, F_{s}^{i} U_{s}^{n}$ is a continuous process integrable with respect to $M_{s}^{i}$, then we have that $U^{n+1}$ is a continuous process on $\mathcal{D}_{M A D}(C)$ with the same property as $U_{t}^{n}$. It follows by induction that $U_{t}^{n}$ is strongly continuous on $\mathcal{D}_{M A D}(C)$ for all $n \in \mathbb{N}$.

We will prove now the basic estimate:

$$
\left\|U_{t}^{n} \xi\right\|^{2} \leq \max _{\eta \in J(\xi)}\|\eta\|^{2} \cdot\left\|U_{0}\right\|^{2} \cdot|J(\xi)|^{n} K_{T}^{n} c_{T, \xi}^{n}\left(\mu_{\xi}(0, t)\right)^{n} \frac{1}{n !}
$$

For all $n \in \mathbb{N}$ and all $0 \leq t \leq T$, where:

$$
c_{T, \xi}=4 \max _{i \in\{1,2,3,4\}, \eta \in J(\xi)} c_{T, i, \eta} \quad, \quad J(\xi)=\bigcup_{i} J_{i}(\xi), K_{T}=\sup _{s \in[0, T]}\left\|F_{s}\right\|_{\infty} .
$$

Using the scalar type integrator inequalities (3.3), (4.4)and (4.5), we have for each $i \in\{1,2,3,4\}$ :

$$
\left\|\int_{0}^{t} F_{s}^{1} U_{s}^{n} a_{s} d s \xi\right\|^{2} \leq\left|J_{1}(\xi)\right|\left(c_{T, 1, \eta}\right)^{2} \sum_{\eta \in J_{1}(\xi)} \int_{0}^{t} d s\left\|F_{s}^{1} U_{s}^{n} \eta\right\|^{2}
$$




$$
\begin{gathered}
\left\|\int_{0}^{t} a_{s}^{+} F_{s}^{2} U_{s}^{n} d s\right\|^{2} \leq c_{T, 2, \eta} \sum_{\eta \in J_{2}(\xi)} \int_{0}^{t} d s\left\|F_{s}^{2} U_{s}^{n} \eta\right\|^{2} \\
\left\|\int_{0}^{t} a_{s}^{+} F_{s}^{3} U_{s}^{n} a b_{s} d s\right\|^{2} \leq c_{T, 3, \eta} \sum_{\eta \in J_{3}(\xi)} \int_{0}^{t} d s\left\|F_{s}^{3} U_{s}^{n} \eta\right\|^{2} \\
\left\|\int_{0}^{t} F_{s}^{4} U_{s}^{n} d s\right\|^{2} \leq c_{T, 4, \eta} \sum_{\eta \in J_{4}(\xi)} \int_{0}^{t} d s\left\|F_{s}^{4} U_{s}^{n} \eta\right\|^{2}
\end{gathered}
$$

where $c_{T, i, \eta}, J_{i}(\xi), i=1,2,3,4$ are defined as in (3.3), (4.4)and (4.5). Then

$$
\begin{aligned}
\left\|U^{n}(t) \xi\right\|^{2} & =\left\|\int_{0}^{t} d M_{t_{1}}^{i} F^{i}\left(t_{1}\right) U_{t_{1}}^{n-1} \xi\right\|^{2} \leq c_{T, \xi} K_{T} \sum_{\xi_{1} \in J(\xi)} \int_{0}^{t} d t_{1}\left\|U_{t_{1}}^{n-1} \xi_{1}\right\|^{2} \\
& =c_{T, \xi} K_{T} \sum_{\xi_{1} \in J(\xi)} \int_{0}^{t} d t_{1}\left\|\left(\int_{0}^{t_{1}} d M_{t_{2}}^{i} F_{t_{2}}^{i} U_{t_{2}}^{n-2}\right) \xi_{1}\right\|^{2}
\end{aligned}
$$

where:

$$
c_{T, \xi}=4 \max _{i \in\{1,2,3,4\}, \eta \in J(\xi)} c_{T, i, \eta} ; J(\xi)=\bigcup_{i} J_{i}(\xi), K_{T}=\sup _{s \in[0, T]}\left\|F_{s}\right\|_{\infty}
$$

because of the conditions on $F^{i}$, then:

$$
\left\|U^{n}(t) \xi\right\|^{2} \leq c_{T, \xi} K_{T} \sum_{\xi_{1} \in J(\xi)} \int_{0}^{t} d t_{1}\left\|\int_{0}^{t_{1}} d M_{t_{2}}^{i}\left(F_{t_{2}}^{i_{2}} U_{t_{2}}^{n-2}\right) \xi_{1}\right\|^{2}
$$

Using the same inequality again

$$
\leq c_{T, \xi}^{2} K_{T}^{2}|J(\xi)| \sum_{\xi_{2} \in J(\xi)} \int_{0}^{t} d t_{1} \int_{0}^{t_{1}} d t_{2}\left\|U_{t_{2}}^{n-1} \xi_{2}\right\|^{2}
$$

An n-fold iteration of the same arguments gives us the estimate:

$$
\begin{aligned}
\left\|U^{n}(t) \xi\right\|^{2} & \leq c_{T, \xi}^{n} K_{T}^{n}|J(\xi)|^{n-1} \sum_{\xi_{n} \in J(\xi)} \int_{0}^{t} d t_{1} \int_{0}^{t_{1}} d t_{2} \cdots \int_{0}^{t_{n-1}} d t_{n}\left\|U_{0} \xi_{n}\right\|^{2} \\
& \leq \max _{\eta \in J(\xi)}\|\eta\|^{2} \cdot\left\|U_{0}\right\|^{2} c_{T, \xi}^{n} K_{T}^{n}|J(\xi)|^{n} t^{n} \frac{1}{n !}
\end{aligned}
$$

Therefore the series $\sum_{n=0}^{\infty} U_{t}^{n}$ converges in the strong topology on $\mathcal{D}_{M A D}(C)$ uniformly on bounded intervals of $\mathbb{R}_{+}$. This implies that it defines a process $U_{t}$ strongly continuous on $\mathcal{D}_{M A D}(C)$. We show that $U_{t}$ is a solution of (5.5). By the integrator of scalar type estimate we have, for all $n \in \mathbb{N}$,

$$
\left\|\int_{0}^{t} F_{s}^{i} U_{s}^{n} d M_{s}^{i} \xi-\int_{0}^{t} d M_{s}^{i} F_{s}^{i} \sum_{k=0}^{n} U_{s}^{k} \xi\right\|^{2} \leq c_{T, \xi} K_{T} \sum_{\eta \in J(\xi)} \int_{0}^{t} \sum_{k=n+1}\left\|U_{s}^{k} \eta\right\|^{2} d s
$$

then, we obtain for all $s \in[0, t], k \in \mathbb{N}, \eta \in J(\xi)$

$$
\left\|U_{s}^{k} \eta\right\|^{2} \leq \max _{\eta \in J(\xi)}\|\eta\|^{2}\left\|U_{0}\right\|^{2}|J(\xi)|^{k} K_{T}^{k+1} c_{T, \xi}^{k} t^{k} \frac{1}{k !}
$$

then the series $\sum_{k=0}^{\infty}\left\|U^{k}(s) \eta\right\|^{2}$ converges in the strong topology on the Fock space uniformly on bounded intervals of $\mathbb{R}_{+}$. It follow from Lebesgue theorem that

$$
\lim _{n \rightarrow \infty} \int_{0}^{t} d M_{s}^{i}\left(F_{s}^{i} \sum_{k=0}^{k=n} U_{s}^{k}\right)=\int_{0}^{t} d M_{s}^{i} F_{s}^{i} U_{s}
$$


This together with the identity

$$
\sum_{k=0}^{k=n+1} U_{t}^{k} \xi=U_{0} \xi+\int_{0}^{t} d M_{s}^{i}\left(F_{s}^{i} \sum_{k=0}^{k=n} U_{s}^{k}\right) \xi
$$

implies that $U_{t}$ verifies the stochastic differential equation (5.5).

ii) uniqueness It will be sufficient to prove that all bounded continuous process $Z_{t}, t \in \mathbb{R}_{+}$satisfying the following white noise stochastic differential equation:

$$
Z_{t}=\int_{0}^{t} F_{s}^{i} Z_{s} d M_{s}^{i}
$$

must be zero. In fact, for all $\psi \in \mathcal{D}_{M A D}(C)$, applying our estimates, we have:

$$
\left\|Z_{t} \psi\right\|^{2} \leq c_{T, \psi} K_{T} \sum_{\eta \in J(\psi)} \int_{0}^{t}\left\|Z_{s} \eta\right\|^{2} d s
$$

applying again the estimates to the integral in the right-hand side $(\mathrm{n}-1)$ times and computing the iterated integral as we did before, we obtain:

$$
\left\|Z_{t} \psi\right\|^{2} \leq \sup _{s \leq T}\left\|Z_{s}\right\|_{\infty}^{2} \max _{\eta \in J(\psi)}\|\eta\|^{2}|J(\psi)|^{n} K_{T}^{n} c_{T, \psi}^{n} t^{n} \frac{1}{n !}
$$

Since this is true for all $n \in \mathbb{N}$, it follows that $Z_{t} \psi=0$ for all $t \in[0, T]$.

\section{REFERENCES}

[1] L.Accardi, F.Fagnola and J.Quaegebeur: A Representation Free Quantum Stochastic Calculus, A representation free Quantum Stochastic Calculus, Journ. Funct. Anal. 104 (1) (1992) 149-197 Volterra preprint N. 18 (1990).

[2] L. Accardi, F. Fagnola: Stochastic Integration, in "Quantum Probability and Aplication III, Lecture Notes in Math, Vol. 1303, pp, 6-19, Springer-Verlag, New York, (1988).

[3] L.Accardi, I.V.Vlovich and Y.G.Lu: A White Noise Approach to Classical and Quantum Stochastic Calculus, Volterra Preprint 375, Rome, July 1999.

[4] L.Accardi: Quantum Probability: An Introduction to Some Basic Ideas and Trends, Modelos Estocasticos II 16, Sociedad Mathematica Mexicana, 2001.

[5] Accardi L., Quaegebeur J.: Ito algebras of Gaussian quantum fields, Journ. Funct. Anal. 85 (1988) 213-263

[6] L.Accardi, Y.G.L.u, I.V.Volovich: Quantum theory and its stochastic limit. Springer Verlag (2002)

[7] Accardi L., Lu Y.G., Volovich I.: Nonlinear extensions of classical and quantum stochastic calculus and essentially infinite dimensional analysis, in: Probability Towards 2000; L. Accardi, Chris Heyde (eds.) Springer LN in Statistics 128 (1998) 1-33 Proceedings of the Symposium: Probability towards two thousand, Columbia University, New York, 2-6 October (1995)

[8] L. Accardi, W. Ayed and H. Ouerdiane: White Noise Flows, in preparation.

[9] S. Attal and J. M. Lindsay: Quantum Stochastic Calculus with maximal operator domains. The annales of probability, (2004), Vol 32.

[10] Ph. Biane: Calcul Stochastique non-commutatif, Séminaire de Probabilités XXIX. Lecture Note in Math(1608), Springer, Berlin.

[11] I. Gelfand and N. Vilenkin: Generelized functions vol.1. Academic Press.

[12] T. Hida: Brownian Motion. Springer, 1992.

[13] Hudson, R. L. and Parthasarathy, K. R.: Quantum Itô's formula and stochastic evolution, Comm. Math. Phys 93(1984), 301-323.

[14] H. Kuo: White Noise Distribution Theory. C.R.C Press, Boca Raton, New York, London, Tokyo, (1996).

[15] K. R. Parthasarathy: An Introduction to Quantum Stochastic Calculus.(1992)Birkhäuser Verlag. Basel.Boston. Berlin.

[16] P. A. Meyer: Quantum Probability for Probabilists, (1993), Springer, Berlin.

[17] L. Schwartz: Theorie des distributions. Hermann, Paris.

[18] K. Yosida: Functional Analysis. Springer, (1978). 